DES2 013859

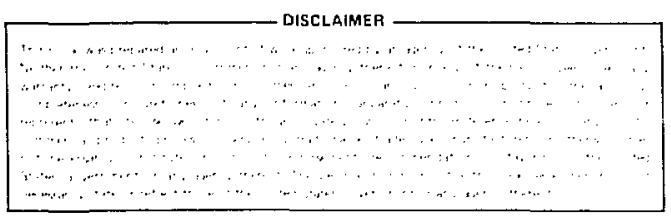

\title{
Thomson-Scattering Systems on TMX
}

\author{
R. K. Goodman \\ J. F. Clauser \\ A. M. Frank \\ D. A. Goerz \\ D. D. Lang
}

Manuscript date: January 30, 1982 


\title{
Thomson-Scattering Systems on TMX
}

\begin{abstract}
This report summarizes the criteria and designs that were used for the two TMX Thomson-scattering systems. It describes the optical, mechanical, electronic, and computer analysis features of these installations. A brief discussion of general Thomson-scattering principles and sensitivity limits is given. Also included are some plasma electron temperature and density measurements from TMX that were obtained through the use of these systems.
\end{abstract}

\section{INTRODUCTION}

Electron temperature $T_{c}$ is one of the most important parameters that characterize a plasma. Its measurement is necessary to describe plasma operating conditions such as: energy loss rates, power balance, plasma stability, and particle transport. It is also used to determine the rate coefficients for spectroscopic analysis. At the present time Thomson scattering is the most accurate and useful methou of measuring $T_{0}$ in a plasma. The advantages of this technique are: (1) it can give a direct measurement of electron temperature $T_{\text {c }}$. and also electron density $n_{6}$, in a small region of the plasma at a specified time, (2) it provides both spatial and temporal resolution of $T_{c}$ and $n_{0}$, and (3) it does not significantly perturb the plasma under most conditions. In recent years Thomson scattering has become a standard diagnostic technique for many plasma experiments. ${ }^{1-15}$
The TMX (tandem mirror) plasma experiment ${ }^{10,17}$ at LLNL has a long central-solenoid cell terminated by minimum-B-type mirror coils (baseball) that plug each end (Fig. 1). High-density plasmas are maintained in the end plugs by neutralbeam injection at high energies. A plasma with lower energy and density is contained in the solenoid. Since the plasmas in the end plugs and the central cell are different, it is desirable to measure $T_{6}$ in each region. Therefore, two independent Thomson scattering systems, one in an end plug and one in the central cell, were installed on TMX (Fig. 2). The design of the system is based on experience gained from operating the Thomson scattering system ${ }^{5}$ on the 2 XIIB plasma experiment.

\section{PRINCIPLES AND GENERAL CONSIDERATIONS}

\section{A. Theory}

The Thomson scattering technique ${ }^{18,19}$ uses a laser beam of monochromatic $\lambda_{i}$ photons that is directed through the plasma. A small fraction of these photons interact with the plasma electrons and are scattered. The cross section for this Thomson scattering is small $\left(o_{1}=0.66 \times 10^{-25} \mathrm{~cm}^{2}\right)$, therefore, an intense, well-collimated beam from a laser is required to provide a sufficient number of photons that are scattered by an angle $O$ (the angle between the laser beam and the center of the viewing optics). Because the plasma electrons have thermal motion, the scattered photons are shifted from $t$ ie laser wavelength by the Doppler effect. An analysis of this wavelength shift can give information on the velocity of plasma electrons. 

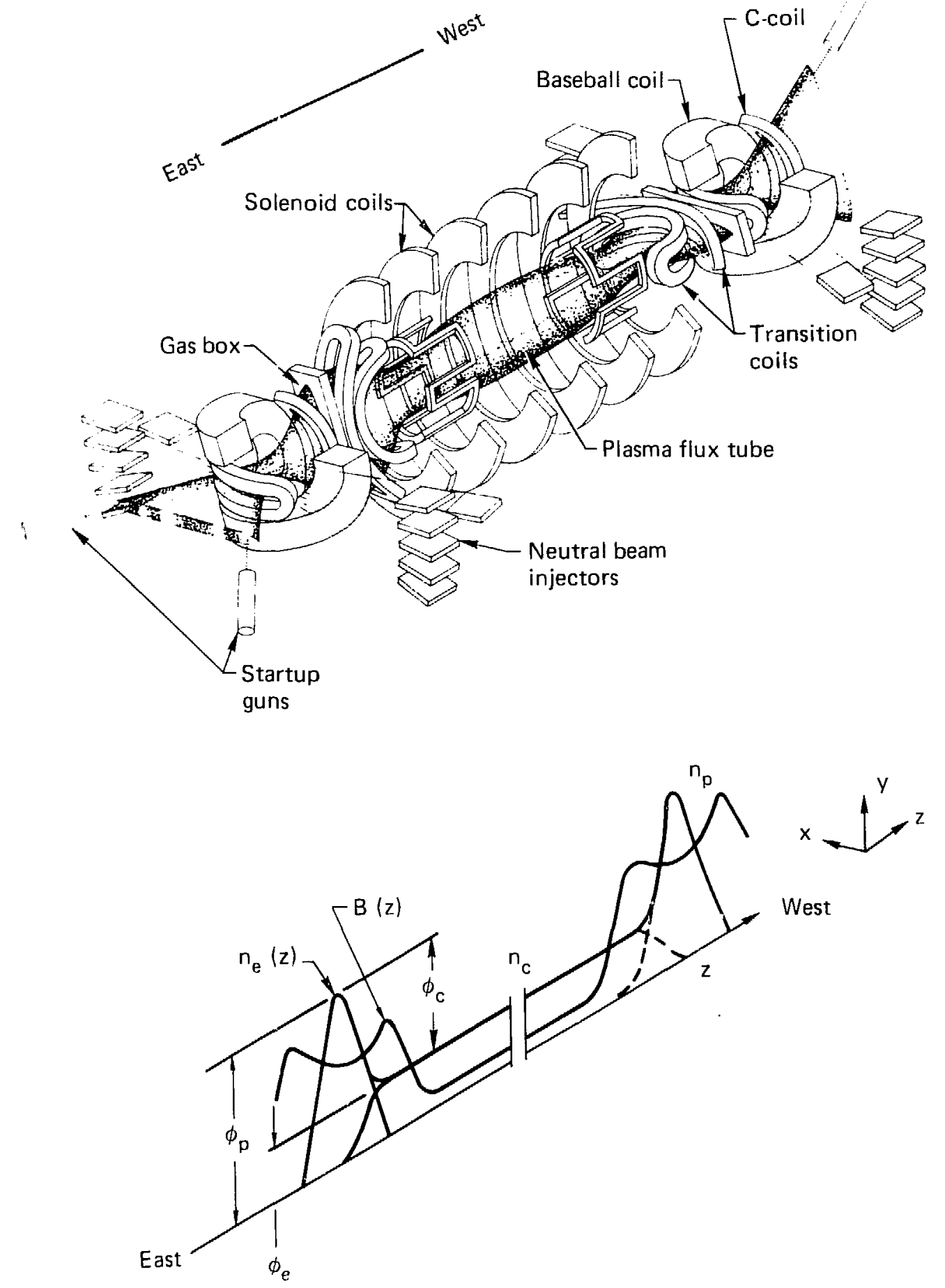

Fig. 1. TMX (Tandem Mirror Experiment) magnet geometry and axial profiles. 


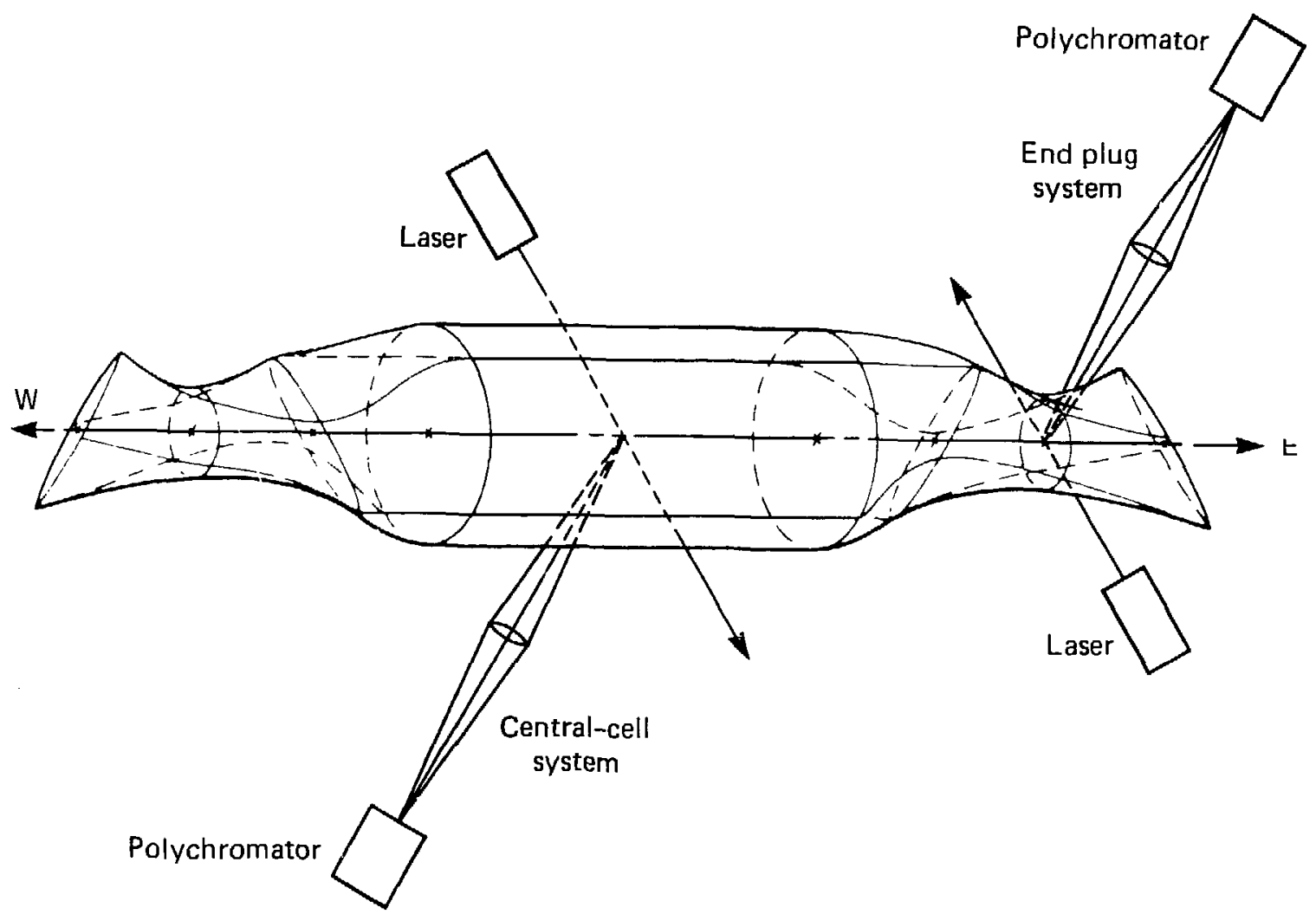

Fig. 2. Schematic diagram showing the location of the TMX Thomson-scattering systems with respect to the magnetic field lines.

If the scattering wavelength $\lambda$ is much less than the Debye wavelength $\lambda_{D}$, the electrons are scattered by uncorrelated electron motion. This condition can be expressed by

$$
\alpha \equiv 1 /|\vec{k}| \lambda_{D}<1,
$$

where $\vec{k}=\vec{k}_{i}-\vec{k}_{s}$, the wave-number shift (Fig. 3). The subscripts $i$ and $s$ refer to incident and scattered wave numbers, respectively.

When this is satisfied, the Doppler-shifted wavelength profile represents the electron velocity distribution in the direction of $\vec{k}$. If $a \ll c$, then

$$
\lambda \simeq \lambda_{1}|2 \sin (0 / 2)|^{-1}
$$

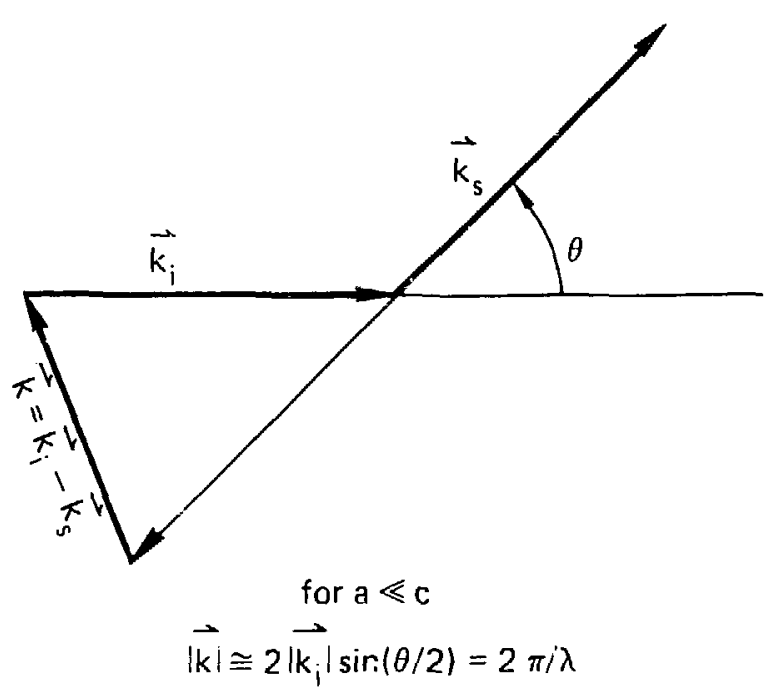

Fig. 3. Wavenumber vector diagram for Thomson scattering. 


$$
\begin{aligned}
a & =\frac{1.08 \times 10^{-1} \lambda_{i}(\mathrm{~cm})}{\sin (\theta) 2)} \\
& =\left[\frac{n_{\mathrm{e}}\left(\mathrm{cm}^{-3}\right)}{T_{\mathrm{e}}(\mathrm{e})}\right]^{12}
\end{aligned}
$$

in TII , when

$$
\begin{aligned}
& 10 \leq T_{e} \leq 300 \mathrm{eV} \text {. } \\
& 1: 10^{12} \leq \mathrm{n}_{\mathrm{e}} \leq 3 \times 10^{13} \mathrm{~cm}^{-3} \text {, } \\
& \theta=100^{\circ} \text {, and } \\
& \Lambda_{1}=0043 \AA \text { (using a ruby laser) }
\end{aligned}
$$

then

$$
c y=<0.02
$$

satisfying the above requirement.

For a plasma with a Maxwellian electron velocity distribution (most plasmas) and a low electron temperature $(<1 \mathrm{keV})$, the observed scattered wavelength profile will be a simple Gaussian with its peak at the laser wavelength. It will be of the form ${ }^{10}$

$$
\begin{aligned}
P_{s}\left(\lambda_{s}\right) d \Omega d \lambda_{s} & =\frac{P_{i} L r_{0}^{2} n_{e} c}{2 a \lambda_{i} \sin (\theta / 2) \bar{\pi}} \\
& x \exp \left[-\frac{(\Delta \lambda)^{2} c^{2}}{4 a^{2} \lambda_{i}^{2} \sin ^{2}(\theta / 2)}\right] \\
& \times d \Omega d \lambda_{5},
\end{aligned}
$$

where

$P_{5}$ is the scattered power,

$\Omega$ is the scattering solid angle intercepted by the viewing optics,

$\lambda_{5}$ is the wavelength of the scattered light,

$P_{i}$ is the laser power,

$L$ is the viewed length of the laser beam,

$r_{0}$ is the classical electron radius,

$\Delta \lambda$ is $\lambda_{s}-\lambda_{i}$.

The halfwidth of this profile at the $1 / \mathrm{e}$ amplitude point is given by

$$
\begin{aligned}
\Delta \lambda_{1 / \mathrm{e}}(A) & =3.95 \times 10^{-3} \\
& , \lambda_{1}(A) \sin (\theta / 2)\left|T_{\mathrm{e}}(\mathrm{eV})\right|^{1 / 2}
\end{aligned}
$$

The total number of photons $N_{3}$ scattered into $\Omega$ can be obtained by integrating Eq. (4) to give

$$
N_{s}=W_{1} r_{0}^{2} n_{e} L ! ! / h v_{i}
$$

where $W_{i}$ is the laser energy, $h$ is Planck's constant, and $v_{i}$, is the laser frequency.

From Eqs. (5) and (6) it can be seen that the width of the scattered spectrum is a direct function of $T_{e}$ and the total collected intensity is proportional to $n_{e}$. For a plasma with a high electron temperature $(>1 \mathrm{keV})$, relativistic effects must be included and the profile of the scattered spectrum is altered. ${ }^{\text {o- }} 21$

\section{B. Measurement}

The profile of the scattered spectrum is determined by separating the light collected by the viewing optics into wavelength channels. This separation is done with either a diffraction grating (polychromator) or a series of optical bandpass filters. In each of these channels the number of collected photons is measured by a photomultiplier (PM) tube or equivalent detector. Relative gains, from the $P M$ tubes and amplifiers, are determined by a calibration; the signals from each channel are then normalized to the same gain. The normalized output from the PM tubes has the same profile as the power spectrum of the scattered light |Eq. (4)|. These output data are sent to a compi'ter that performs the normalization and calculates the $T_{e}$. The data can also be reduced by hanu from oscilloscope traces of the PM tube signals. To do this, the logarithm of the normalized signal from each channel is plotted versus $(\Delta \lambda)^{2}$ and $\mathrm{Eq} .(5)$ is used to determine $T_{\mathrm{e}}$ (Fig. 4). If the total system has been calibrated for density (Section II-D), $\mathrm{n}_{\mathrm{e}}$ can also be obtained from these data either by the computer or by hand.

\section{SYSTEM SENSITIVITY}

The output of a photomultiplier tube depends on the number of si attered photons $N_{s c}$ received by that channel and the quantum efficiency $\eta$ of the phoiocathode. Since the conversion from photons to photoelectrons is governed by Poisson statistics this may be written as ${ }^{19}$

$$
N_{\mathrm{se}}=\mathrm{N}_{\mathrm{SC}} \eta \pm\left(\mathrm{N}_{\mathrm{sc}} \eta\right)^{1 / 2}
$$






Fig. 4. Plot of signal amplitude vs wavelength shift for various electron temperatures. Also shown are the PM channel wavelength assignments for the TMX Thomson-scattering systems.

where $N_{50}$ is the number of photoelectrons emitted. The signal-to-noise ratio for a channel is then

$$
S \mathbb{N}=N_{, 0} \cdot\left(N_{s e}\right): \cdots\left(N_{,} \eta\right)^{\prime}=
$$

Thus, from Eqs. (o) and (8), S/N can be improved by: (1) increasing the laser power, (2) increasing the plasma density, (3) improving the transmission of the viewing optics, and (4) using PM tubes with a higher quantum efficiency. The accuracy of Thomson scattering data and the resulting practical measurement limit are determined by SIN.

From Eq. (0) and the TMX parameters |10-I ruby laser, $L=1 \mathrm{~cm}, \Omega=0.033 \mathrm{sr}$ (with $\mathrm{f} / 4.9$ viewing optics) and $\mathrm{n}_{e}=1 \times 10^{13} \mathrm{~cm}^{-3} \mid$, the expected total number of collected photons is $N_{s}=9.5 \times 10^{5}$. This agrees with the magnitude of the signals that we observe, and shows that the overall sensitivity is usually not the limit of our ability to make measurements.

In addition to the Thomson scattered photons, the viewing optics also collect photons from plasma radiation. This includes Bremsstrahlung, recombination, and line radiation. An estimate of the expected number of photons from Bremsstrahlung $N_{B}$ gives $N_{B}<10^{-3} N_{5}$, which is negligible. However, on both the 2XIIB and TMX experiments we have observed that the ratio of $N_{5}$ to $N_{p}$ (the number of plasma radiation photons collected) ranges between :: o and ten depending upon the impurity condisuns in the experiment. This is similar to what had been observed on the T3-A experiment. ${ }^{3}$

The amount of plasma radiation present places limits on the ability of a Thomson scattering system to make measurenents. When plasma radiation is included, Eq. ( 8 ) becomes

$$
S / N=\frac{\left(N_{\mathrm{sc}} \eta\right)^{1 / 2}}{\left(1+\frac{N_{\mathrm{pc}}}{N_{\mathrm{sic}}}\right)^{1 / 2}}
$$

where $N_{p t}$ is the number of plasma radiation photons received by a PM channel. Therefore, the presence of plasma radiation in a channel sets an effective sensitivity limit for that channel. Also, a strong $\mathrm{N}_{2}$ line at $6468 \AA$ in TMX (Fig. 5) rendered one of our photomultiplier channels unusable. For the TMX plasmas some plasma radiation appcared 


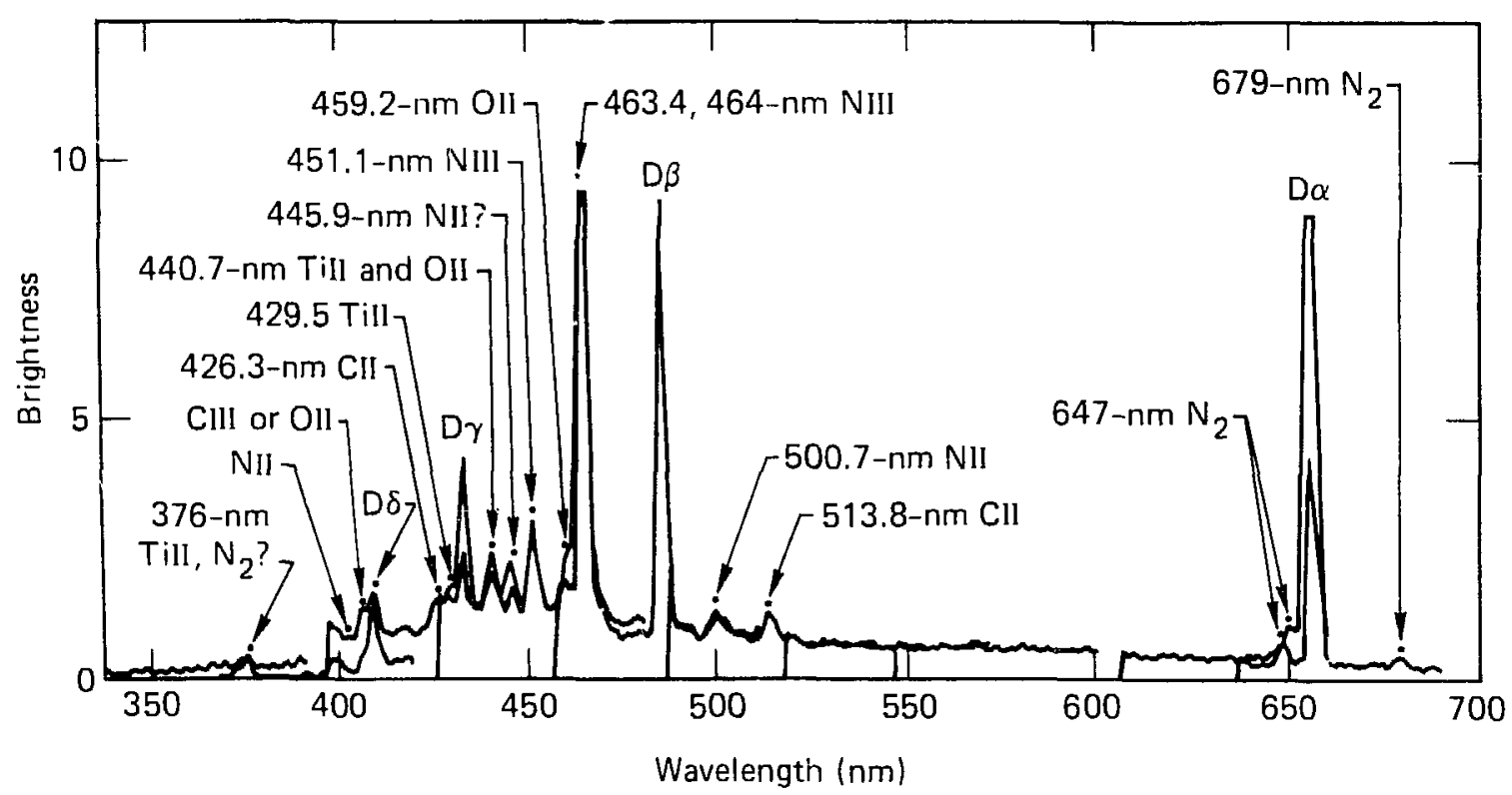

Fig. 5. Impurity line spectra from TMX central-cell plasma over a period of two days.

in most of the PM channels and was the limiting factor on our ability to make measurements. Because the scattered radiation is plane polarized, while the plasma radiation is not, an improvement in the $N_{s}$ to $N_{p}$ ratio oy a factor of 2 can be obtained by using a polarizing element in the vicwing optics. Also, $N_{s} / N_{F}$ is inversely proportional to the plasma diameter since the viewing optics receive plasma radiation generated along a chord through the plasma while the scattering volume is constant. Since $N_{p}$ is approximately constant when the plasma is in a state of equilibrium, the level of $N_{p}$, as seen by each PM tube, can be sampled near the time of the scattering measurement and can be subtracted from the total signal of that tube.

From Eq. (4) the peak of the scattered power spectrum can be written as

$$
P_{s}\left(\lambda_{s}\right) \propto \frac{P_{i} n_{e} d \lambda_{s}}{\lambda_{i} \sin (\theta / 2) \sqrt{T_{e}}}
$$

Therefore, for channels near the laser wavelength, there is an effective upper limit on $T_{e}$ ( for a given $n_{e}$ and $P_{i}$ ) to keep above a minimum, usable S/N. Figure 6 shows some TMX Thomson scattering $T_{e}$ and $n_{e}$ measurements near a minimal $S / N$. Here the density values have been normalized to a laser energy of $10 \mathrm{~J}$ (to give an equivalent

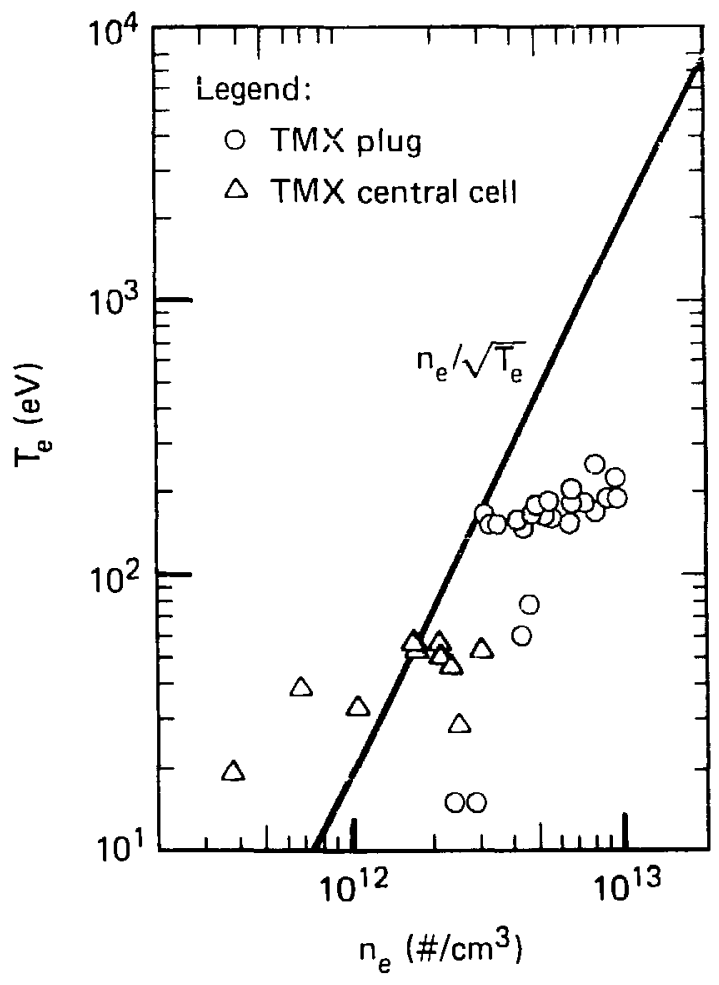

Fig. 6. Thomson-scattering $T_{e}$ and $n_{e}$ measurements from TMX. The line indicates the limit for measurements with the plug system. 
number of photons) since some of these measurements were made with a laser energy of only $3 \mathrm{~J}$. The line is $n_{e}\left(T_{e}\right)^{-1 / 2}$ which is a constant number of photons per unit wavelength at the peak of th.e profile. Assuming a constant plasma radiation level, this line represents the practical sensitivity limit of our measurements with the plug system. Usable parameter space lies to the right of this line, with $S / \mathrm{N}$ also increasing to the right. From Eq. (10) this line could be moved to the left either by increasing the laser power or by lowering $\lambda_{i}$ or $\theta$. $\therefore$ smaller $\theta$ can also improve $N_{s} / N_{p}$ as it compresses the scattered spectrum in $\Delta \lambda$ while the plasma radiation spectrum remains constant. The central-cell points (Fig. 6) that lie to the left of the line show the effect of using PM tubes with a higher quantum efficiency. The plug system had PM tubes with quantum efficiencies from 2 to $4 \%$; PM tubes of the central-cell system had quantum efficiencies from 10 to $20 \%$.

Another possible limit on sensitivity is the amount of stray laser light reflected into the viewing optics and seen by the PM tubes. This is more of a concern with channels near the laser wavelength and depends upon the rejection efficiency of the polychromator or filter system. However, careful design of the laser optics (Sections VI-B and -C) can minimize this problem. For our systems, the channels next to the channel that contained the laser wavelength showed no laser light. Even in the laser wavelength channel the amount of laser light was low enough that it could be subtracted and the signal from that channel could be used in the $T_{e}$ measurement. The amount of stray laser light seen by the various channels can be checked by firing the laser under normal conditions, except with no plasma present.

\section{DENSITY CALIBRATION}

As is shown in Eq. (6), the total number of photons collected is proportional to plasma density. The Thomson scattering system can be calibrated for absolute density by substituting a gas of known density for the plasma and measuring the total light collected. This Rayleigh scattering of light from a gas has the same radiation characteristics as Thomson scattering The ratio of cross sections from Rayleigh and Thomson scattering $\left(\sigma_{R} / \sigma_{T}\right)$ is known for several gasses ${ }^{19}$; nitrogen is the one often used. Because the viewed volume and all of the optics are the same, the ratio of Rayleigh-to-Thomson scattering signal can be expressed as

$$
\frac{N_{R}}{N_{T}}=\frac{W_{i R} n_{g} \sigma_{R}}{W_{i T} n_{e} \sigma_{T}}
$$

where $\mathrm{N}$ is the total light collected, $W_{\mathrm{i}}$ is the laser energy, $n_{g}$ is the gas tensity, and $R$ and $T$ refer to Rayleigh and Thomson scattering, respectively. Since the calibration gas is at room temperature, the Doppler shift will be very sniall and all of the Rayleigh scattered light will be essentially at the laser wavelength. To use this calibration the relative laser energy must be monitored for the calibration and for each plasma measurement.

Both Thomson scattering systems on TMX wcre calibrated by filling the vacuum tanks with $\mathrm{N}_{2}$ to several pressures between 10 and 75 Torr. The total scattered light was measured at each pressure by the PM tube on a channel at the laser wavelength. The calibration pressure must be high enough that the Rayleigh signal is much greater than the stray laser light signal on that channel and also so the absolute pressure can be measured accurately. The pressures we used gave signals that would saturate the PM tube and, therefore, required the use of a calibrated neutral-density filter in the optical chain. This factor was then included in the calibration constant. To minimize the error due to the scattering of light from dust particles, the tanks were allowed to sit overnight at 75 Torr before any calibrations were taken. The tanks were then pumped in steps to give measurements at lower pressures. Any subsequent change in alignment of the optical system or the laser can change the calibration constant by changing either the viewed volume or the optical transmission of the svstem.

\section{BASIC DESIGN CRITERIA}

For the TMX experiment it was desired to know the radial $T_{e}$ and $n_{e}$ profiles in the plug plasmas. Based on our experience with the Thom- sun scattering system on the 2 XIIB experiment, we decided to use conventional optics rather than a more complex system such as that in use at 
Princeton ${ }^{0}$ The viewing optics of the plug system were designed to simultaneously image five $1-\mathrm{cm}$ segments of the laser fath through the plasma. These segments corresponded to plasma radial positions (R) of $-5,0,5,10$ and $15 \mathrm{~cm}$. Because of the ports available on the vacuum tank, the scattering angle $\theta$ was $100.22^{\circ}$ for the $R=0$ position and was slightly different for the other radial positions. The optical system was also designed to utilize three polychromator and PM tube sets to make simultaneous measurements at any three of these radial positions (Fig. 7). Only two polychromators were actually installed. The radial position viewed by each polychromator could be shifted by changing mirrors in the optical path.

The central-cell system was simpler as its optics imaged only the $\mathrm{R}=0$ position and therefure had one polychromator and PM set. The scattering angle of the central-cell system was $89.99^{\circ}$.
These TMX Thomson scattering systems were designed to measure temperatures between 10 and $350 \mathrm{eV}$. The Gaussian spectral profiles corresponding to temperatures in this range are shown in Fig. 4. This type of plot is useful for determining how to divide the expected scattered spectra into wavelength channels. The number and arrangement of the channels for the TMX system was based on experience from 2 XIIB. To calibrate the system for absolute density (Section II-D) one channel was needed on $\lambda_{i}$. For the low temperatures, several narrow channels near the laser wavelength were necessary. To have enough channels for reasonable measurement required channels on both sides of $\lambda_{i}$ for accuracy in this range. Generally channels are placed on the shorter wavelength side since PM tubes have higher quantum efficiencies in this region. For higher temperatures, progressively wider channels were used at larger $\Delta \lambda$ to compensate for the



Fig. 7. Schematic diagram of the TMX plug Thomson-scattering system. 


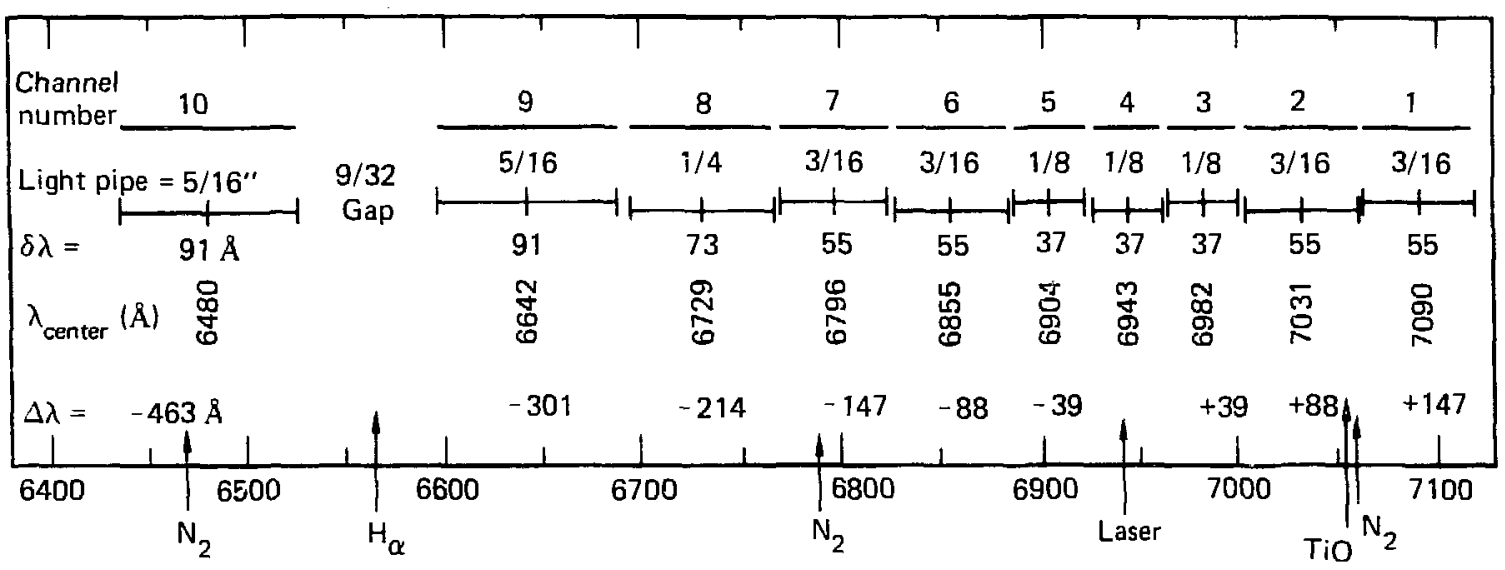

Fig. 8. Photomultiplier channel wavelengths assignments and light pipe dimensions.

lower numbers of scattered photons at these wavelengths. The resulting channel placement is also shown in Fig. 4; the wavelength positions are shown in Fig. 8. A gap was placed around the $D_{a}$ line at $6563 \AA$.

The expected electron temperatures and densities $\left(1 \times 10^{12} \leq \mathrm{n}_{\mathrm{e}} \leq 3 \times 10^{13} \mathrm{~cm}^{-3}\right)$ required the use of the highest energy, pulsed lasers available $\mid$ Eq. (10)|. Ruby lasers $\left(\lambda_{i}=6943 \AA\right)$ with a pulse width of 20 to $40 \mathrm{~ns}$ and energies from 10 to 12 I ar: readily obtained. They are commonly used $i_{i}$ this application and were chosen for our systems. Also, the wavelength of the ruby laser is in a region that is relatively free of strong plasma radiation (Fig. 5). Neodymium glass and YAG lasers $(\lambda=10600 \AA)$, with their higher energies, lie in a wavelength region that is not suitable for PM tubes. Doubled Nd-glass and YAC lasers $\left(\lambda_{1}=5300 \AA\right) 22$ were in the development stage and were not considerer when these systems were being designed.

\section{MECHANICAL DESIGN}

The Thomson scattering system located on the TMX plug (Fig. 9) was composed of two subsystems: the laser beam line components and the scattered-light-viewing components. The overal! configuration (vertical view tube and almost horizontal laser path) was chosen to reduce the light from the neutral-beam injectors (Fig. 1) that would be collected by the viewing system. This arrangement allowed for good accessibility to the various components by placing them on top of the vacuum vessel (Fig. 10).

For overall system stability the laser, beam transport boxes, distribution box, polychromators, and PM tube boxes were all rigidly mounted to the vacuum vessel by a stainless-steel box-beam and I-beam frame. The view tube, laser input arm, and beam dump were mounted directly on the vacuum vessel. The alignment stability of the system was quite good. Realignment was required only after the vacuum vessel was rolled apart for general TMX mairtenance or after an earthquake (January 24, 1980).

The system was designed to allow final alignment of all componenis while TMX was under vacuum. The view tube, mounted on a gimbal and connected to the tank with a bellows, was aligned by jacking screws. Adjustments to the laser input arm and its mirror were by bellows-type vacuum feed throughs. The laser beam dump had a bellows vacuum seal and could translate $\pm 2.5 \mathrm{~cm}$ either vertically or horizontally on linear bearings. It could also be tilted $\pm 5^{\circ}$ in either plane. For alignment purposes, a mechanical flag could be rotated into the center of the plasma region. It defined the main alignment point and both the laser and view tube could be aligned to the same spot on this flag. 


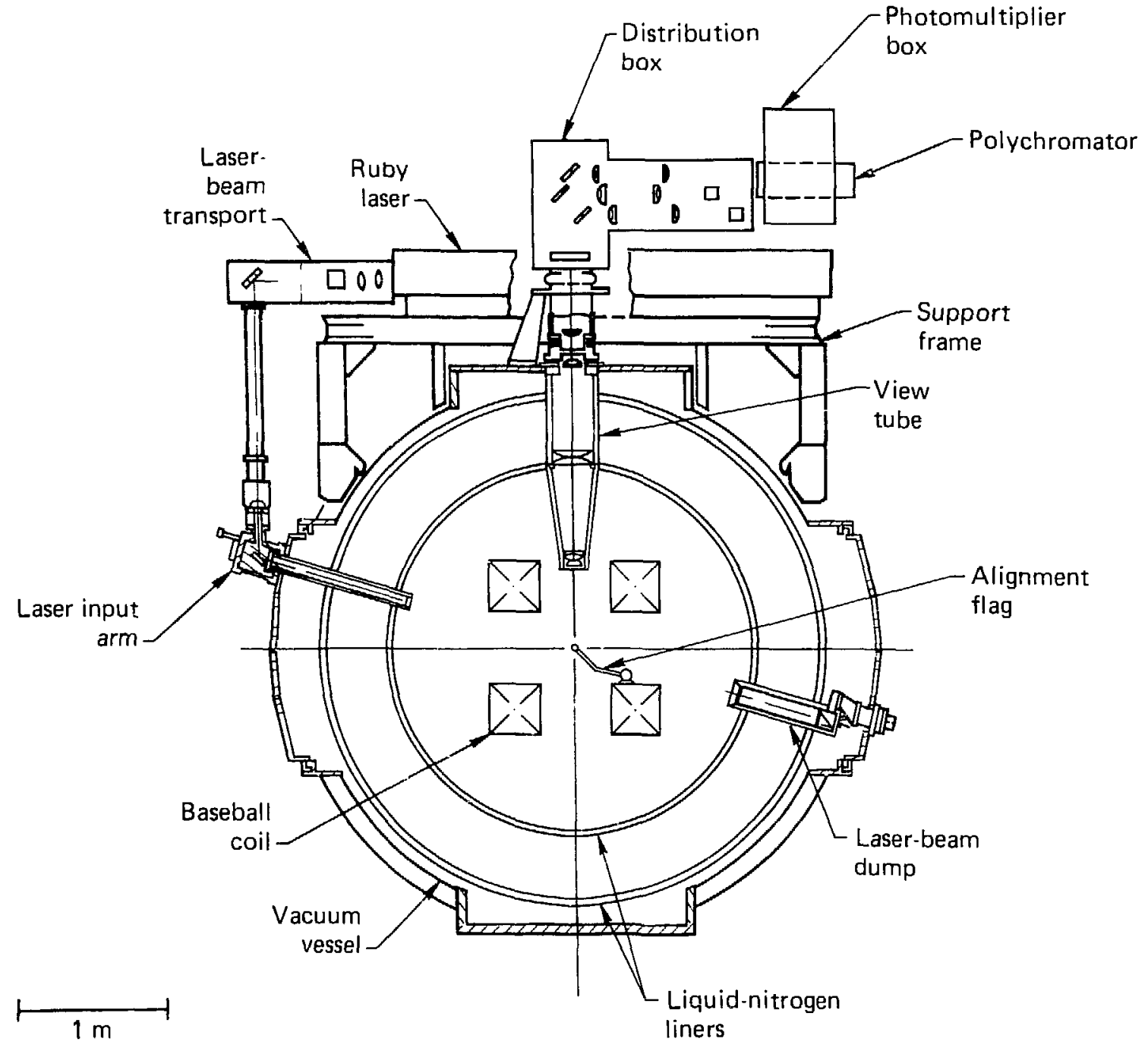

Fig. 9. Location of components for the TMX plug Thomson-scattering system.

All of the components located inside the vacuum system had heat shields to minimize thermal gradients caused by the liquid nitrogencooled liners or the titanium sublimators used for vacuum pumping. The inner ends of the input arm, beam dump, and view tube each had a mechanical shutter to prevent titanium from striking any of the optics. These tight-fitting shutters, open only during a plasma shot, helped to keep the optics clean and maintain their optical efficiency. The interior sections of the input arm, beam dump, and view tube were vented to the main vacuum system through 20 -micron filters in the walls of their solid housings. All of the optics and compcnents of these units were cleaned and assembled in a Class-1000 clean room.

The central-cell Thomson scattering system was similar to the plug system except that the view tube was located on the bottom of the TMX and looked up. Its distribution box, polychromator, and PM tube box were located under the vacium vessel. This was necessary because other diagnostics were located on the top of th ? central cell. The laser and its transport boxes were located on top of the vacuum vessel and the laser path through the plasma was almost horizontal. 




Fig. 10. View of TMX during construction showing the plug Thomson-scattering system. The polychromators and PM tubes are not installed.

\section{LASERS}

\section{A. PLUG SYSTEM}

The commercially-manufactured $Q$-switched ruby laser in the plug system was originally designed to pruduce four $10-1$ pulses during a plasma shot. However, its operation in that mode was never satisfactory and, therefore, it was used as a single-pulse 10- 1 laser. Its pulse width was 20 to 40 ns full width, half maximum (FWHM); the beam divergence was $1.5 \mathrm{mrad}$ full angle, half energy (FAHE) after its beam was expanded to 5 -cm diameter.

The oscillator head had a $15.2-\mathrm{cm}$ by $0.95-\mathrm{cm}-$ diam ruby rod. Its beam diameter was limited to $0.04 \mathrm{~cm}$ by an aperture on the head. The three amplifier heads each had a $12.7-\mathrm{cm}$ by $1.91-\mathrm{cm}-\mathrm{diam}$ rod. To match the diameter of the amplifier rods, the uscillatur beam was expanded by a factor of 3 heture entering the first amplifier. Following the final amplifier the beam was expanded 2.7 times to produce a parallet 5-cm-diam beam. This expansion was done to prevent damage to the turning mirrors in the beam path. On the 2 XIIB system we found that the damage threshold to our mirrors was approximately $1 \mathrm{~J} / \mathrm{cm}^{2}$. Because of the way the amplifier rods were pumped, the expanded beam is hollow. Figure 11 is an imprint from the expanded beam striking a piece of burn paper (photographic paper that has been exposed and developed).

To align the laser beam, a helium-neon (FieNe) laser was introduced into the system through the rear cavity mirror of the oscillator (Fig. 12). Its beam was expanded to match the diameter of the ruby laser beam and could easily be made colinear with the ruby beam by adjusting the two turning mirrors. The oscillator energy was monitored by measuring the fraction of the ruby beam that leaked through the rear cavity mirror with a vacuum photodiode. The energy monitor was calibrated by firing the oscillator and con paring the signal from the photooiode with the signal from a calorimeter placed after the front cavity mirror.

It was found that the TMX magnetic field degraded the operation of the plug laser's ostillator 


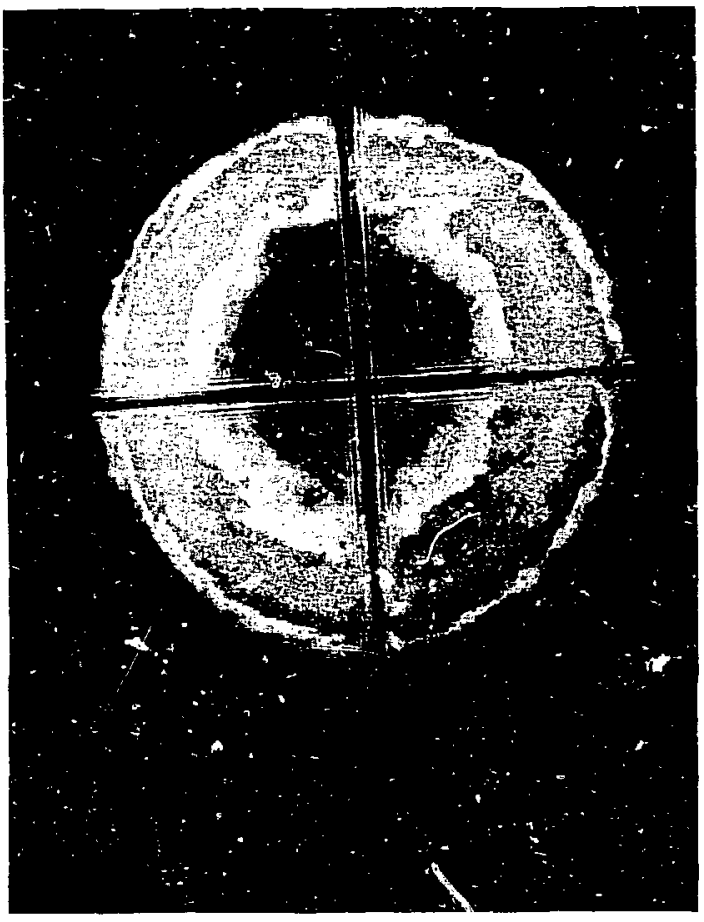

Fig. 11. Full-size burn-paper imprint of the expanded laser beam. The crossed lines are from alignment reticles. head. The field in this region $(-0.02 \mathrm{~T})$ was transverse to the rubv rod. A carbon-steel box around the oscillator head was necessary to prevent this from occurring.

\section{B. CENTRAL-CELL SYSTEM}

The Q-switched ruby laser used in the centralcell system was also manufactured commercially. It was a 12-I single-pulse laser with a pulse width of $25 \mathrm{~ns}$ ( $F W H M$ ) and a beam divergence of $2 \mathrm{mrad}$ (FAHE) at a beam diameter of $1.91 \mathrm{~cm}$. The oscillator head had a $15.2-\mathrm{cm}$ by $1.59-\mathrm{cm}$-diam ruby rod. It had a single amplifier head with a $15.2-\mathrm{cm}$ by 1.91-cm-diam rod. There was no heam expander between the oscillator and amplifier. Following the amplifier, the beam was expanded to 5-cm diameter as in the plug system. The oscillator power monitor and the HeNe alignmerit laser in this system were similar to those used with the plug laser.



Fig. 12. Schematic of the oscillator sertion of the plug laser. Also shown is the helium-neon beamalignment laser. 


\section{BEAM-LINE COMPONENTS}

\section{A. BEAM TRANSPORT}

Figure 13 shows the beam transport components for the plug system. The total energy of the laser was monitored by measuring the small fraction of the beam that leaked through the first urning mirror. This beam sample was diffused by deflecting it from a magnesium-oxide disk before it entered the vacuum photodiode detector. Diffusing the beam sample minimized measurement errors that would be caused by the hollow nature of the main laser beam in this region (Fig. 11). The energy monitor was calibrated for absolute encrgy by firing the laser and comparing the photodiode signal with the signal from a calibrated calorimeter. It ivas the energy measurement from this detector that was used in the plasma density measurements (Section II-D).

Three turning mirrors were required in the bean transport section to preserve the correct polarization (normal to the viewing system axis) of the laser beam as it traversed the plasma. Each of these mirrors was a dielectric interference coating supported on a 1.91-cm by $9.78-\mathrm{cm}$-diam Bk-7 glass substrate. The coating was $99.8 \%$ reflecting for a wavelength of $0943 \AA$ at an incident angle of $45^{\circ}$. These mirrors, along with the other laser beam-line components, were ultra-low scatter (ULS) with $<5 \AA$ RMS surface roughness and $<10.5$ total integrated scattering after coating. The mirrors were mounted on commercial mirror holders and optical rails that allowed both translation and tilt.

Following the second turning mirror were two alignment reticles (Fig. 13) mounted on an optical rail. They were used to define a reference beam axis. The laser beam could be centered on these reticles by adjusting the first and second turning mirrors. A superimposed image of their cross hairs is seen in Fig. 11. The beam could easily be returned to this reference axis after any changes to the laser or its realignment, so realignment of the following components in the laser path was not necessary. This is a very useful feature of the system.

Although more compact, the beam transprort section of the central-cell system was similar to that of the plug section. Because the axis of the central-cell laser was parallel to the TMX axis its transport section contained four turning mirrors tu preserve the correct polarization.

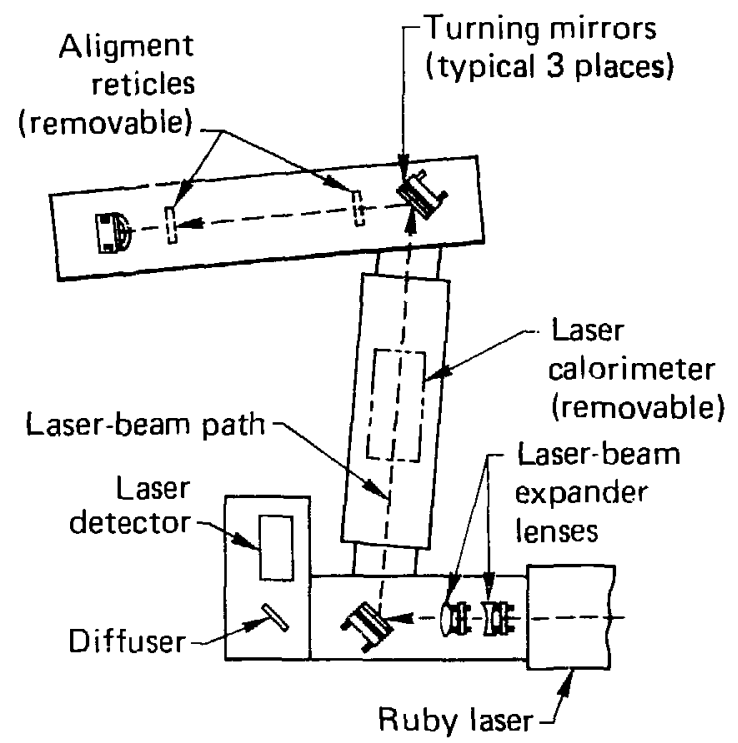

Fig. 13. Layout of the beam-transport section of the plug Thomson-scattering system.

\section{B. INPUT ARM}

The input arm assembly, which was identical in both the plug system and central-cell system, is shown in Figs. 14 and 15. To reduce the number of optical surfaces, the beam lens doulled as a vacuum window. This $9.27-\mathrm{cm}$-diam plano-convex lens fabricated from Bk-7 had antireflection (AR) coatings $(<0.1 \%$ reflectivity) on both surfaces. Its 272-cm focal length gave a narrow cone angle and allowed measurements to be made at various radial points in the plasma. The isulation valve allowed the lens to be changed, if ner "ssary, without taking the main vacuum system io air. The turning mirror was the same as the mirrors in the transport section except for its elliptical shape and different beam input angle.

Details of the beam collimation for the input arm and beam dump are shown in Fig. 10. Each collimator was fabricated from a dye gluss that absorbed radiation in the wavelength region near $0943 \AA$. The sizes of the collimators and their knife edges were designed to reduce the amount of scattered laser beam that could enter the 


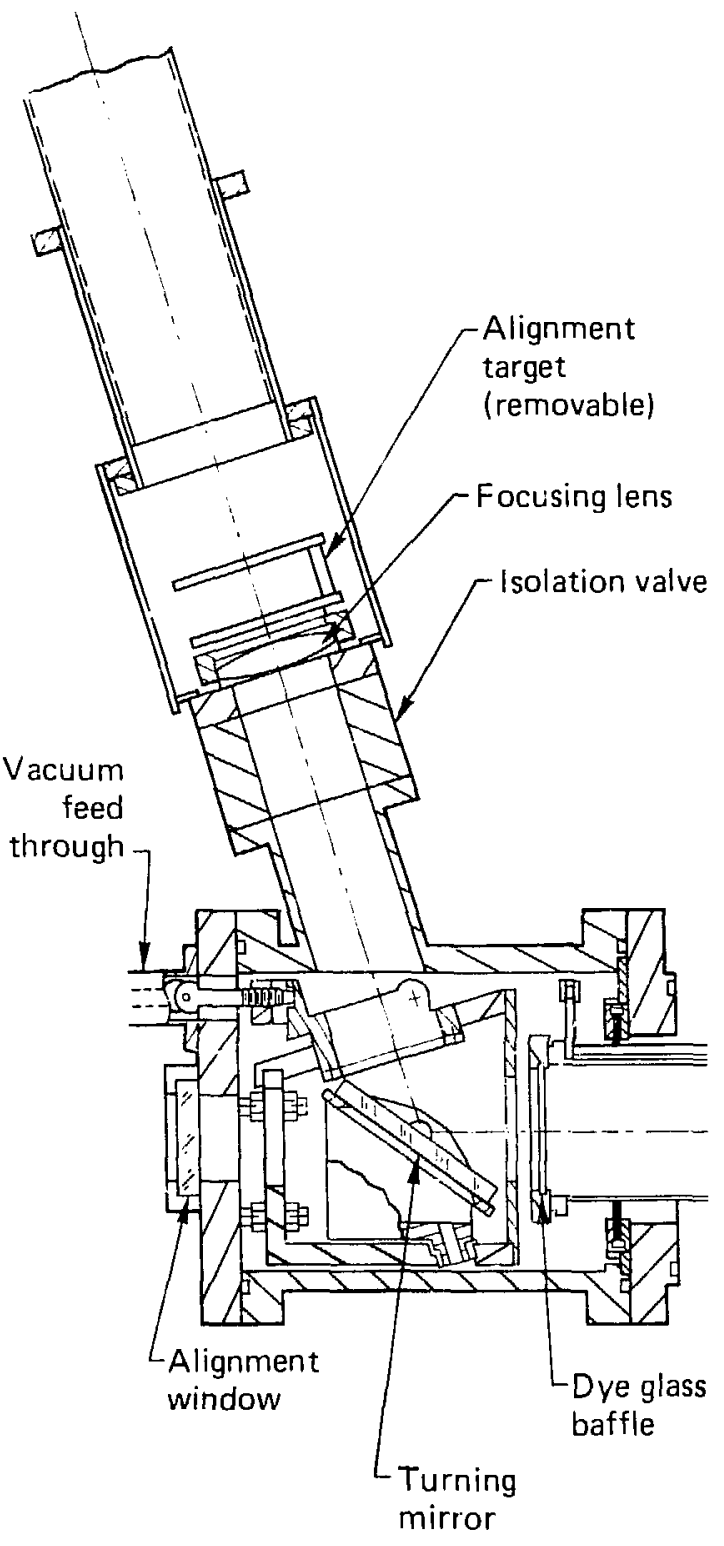

Fig. 14. Laser beam input arm assembly.

viewing optics (Section II-C). Antireflection coatings and the ULS surfaces also contributed to the reduction of beam scattier.

\section{BEAM DUMP}

Details of the laser beam dump, identical in both systems, are shown in Fig. 17. The collimators were similar to those in the input arm. The image of the laser aperture is at the aperture

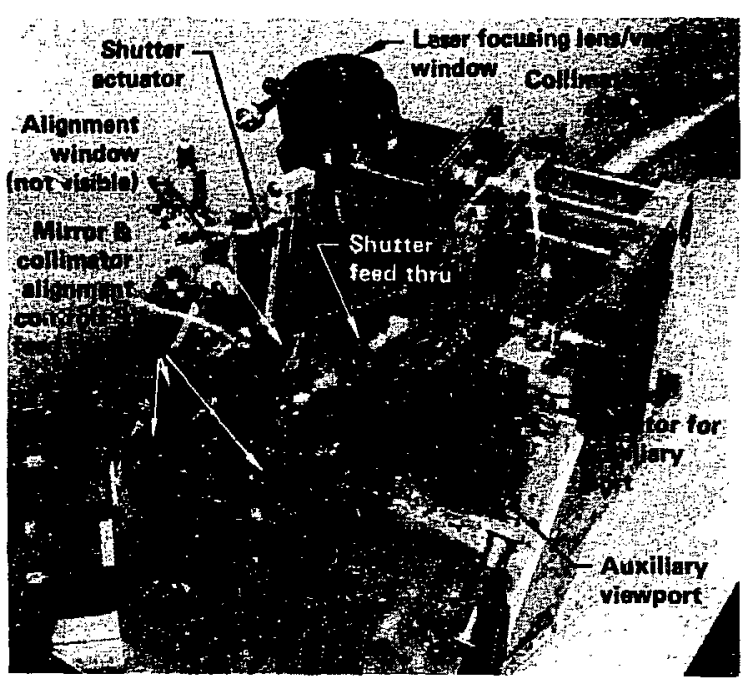

Fig. 15. Laser beam input arm.

of the first dump collimator (Fig. 16) to ensure that all diffracted laser light falls on the dump. The dump pieces were fabricated from the same absorbing dye glass as the collimators. The main dump piece (Fig. 16) was set at Brewster's angle for maximum transmission. The secondary dump absorbed most of the beam that was reflected from the main dump. The total collimating and dump system was very efficient in controlling the laser beam (Section II-C)

The fiber optic bundle, behind the center of the main dump, was connected to a combination photodiode detector and high-intensity lamp unit. The photodiode could be used as a general monitor on the laser alignment by measuring the amount of beam transmitted through the center of the dye glass when the laser was fired. The lamp could also be used to check the laser beam alignment. With the lamp on and one of the alignment reticles installed in the beam transport section, a blue spot (color of the dye glass) could be seen by looking down the laser optical chain from a position near the output of the laser. If the optics were correctly aligned, the cross hairs of the reticle would appear centered on the spot from the dump.

\section{LASER BEAM ALIGNMENT}

The alignment of the laser beam in each system was done with the HeNe laser beam and then checked by firing the ruby laser into a burn 


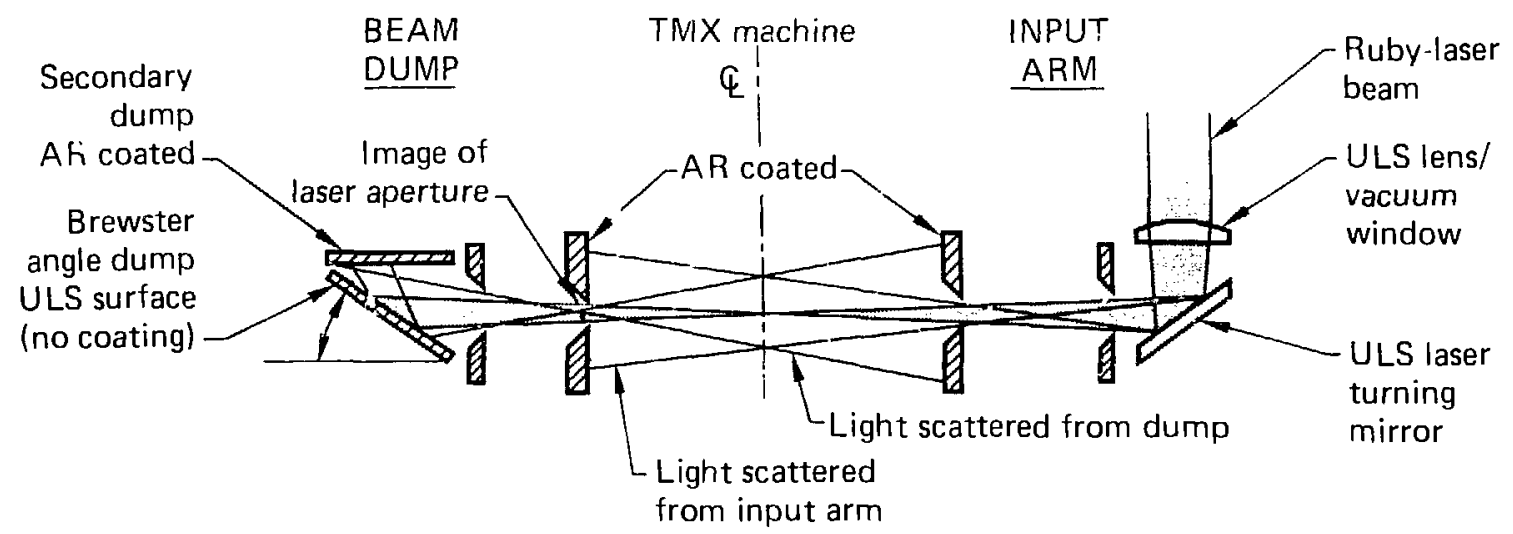

$Q 7$ Dve glass components

Fig. 16. Schematic of the laser beam collimination and dump components.

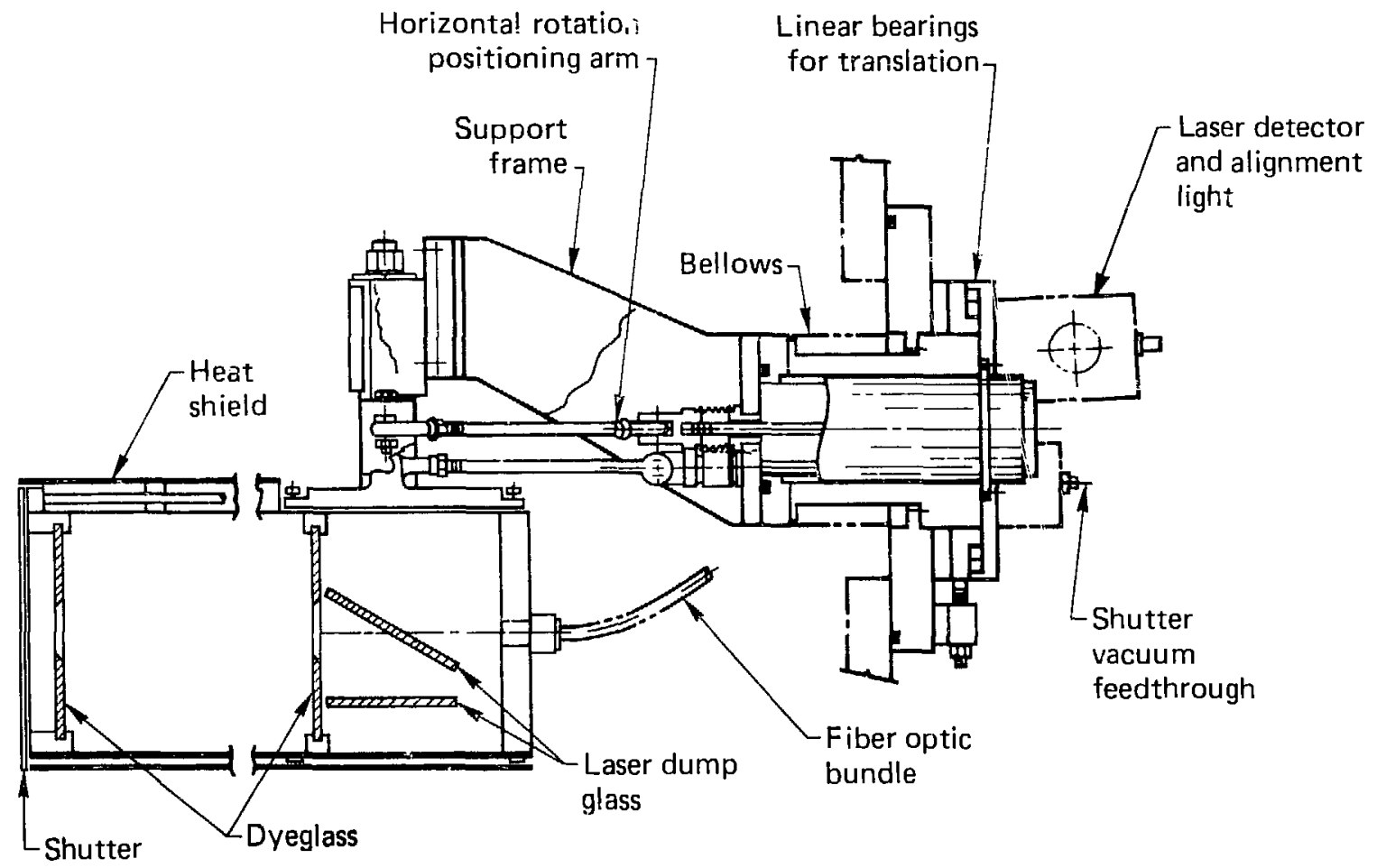

Fig. 17. Laser-beam dump assembly.

paper at various locations. The beam was first centered on the two reticles in the transport section as described above. Then the last turning mirror was adjusted to the center of the beam on an alignmeıt reticle at the focusing lens (Fig. 14).
An alignment telescope looked through a vacuum window and the turning mirror of the input arm. It could view the end of the input arm, the alignment flag, and the beam dump. When TMX was under vacuum, the lignment flag and 
the beam dump could be illuminated by a spotlight shining through an auxiliary window on the input arm assembly (Fig. 15). With this telescope the beam could be centered on the alignment flag by adjusting the rotation (horizontal plene) and tilt (vertical plane) of the input arm mirror. This set the laser path through the plasma. The input arm and the mirror rotated to cot!ier. Their alignment in this plane had been adjusted with a bench setup when the input arm was assembled. The tilt of the mirror and the input arm was independently adjustable. The input arm tilt was aligned by center ing a target, marked on the inner surface of the input arm shutter, on the HeNe beam.

The optical path of the alignment telescope was made colınear with the laser bcam by adjusting the position and tilt of the telescope and its turining mirror (Fig. 15). This telescope was then used to align the beam dump. The dump assembly was translated to center the fiber-optic light spot described above on the telescope axis and was rotated to center the target marked on the dump shutter.

\section{VIEWING AND DETECTION COMPONENTS}

\section{A VIEW TUBE}

The large diameter of the vacuum vessel in the plug region required the use of a series of reldy lenses in the viewing optics. The plug system view tube is shown in Fig. 9; a layout of its optical components is shown in Fig. 18. These optics were designed to image a $20-\mathrm{cm}$ section of the laser path through the plasma. This allowed simultaneous measurements at more than one radial position in the plasma. The strong curvature and astigmatism over the total field was not significant in the 1.5-crr. segments used by each polychromator. The sagital focus was parallel to the entrance slits of the polychromators.

For maximum light input, the objective lens was as large in diameter as oossible $(125 \mathrm{~mm})$ and was placed as near the plasma as practical. It had an input cone of $\mathrm{F} / 4.9$. The first relay lens had a $150-\mathrm{mm}$ diameter to prevent vignetting. The requirem $n \mathrm{n}$ to image points $10 \mathrm{~cm}$ off axis made it necessary to use $300-\mathrm{mm}$-diam field lenses. To minimize aberrations, the vacuum window was placed between the last pair of relay lenses. The output cone of this assembly was made $f / 6$ to match the polychromator input optics. The view tube uptics had a magnification factor of 1.5.

All the lenses were commercially available and, except for the field lenses, were air-spaced acromats. Both surfaces of each optical component were AR coated ( $<0.3 \%$ reflection in a broad band). The total transmission of this assembly with 22 optical surfaces was quite good (Fig. 19).

The viewing optics of the central-cell system were designed to image only one position in the plasma. Because of this and the smaller diameter of the vacuum vessel, a single air-space acromat was used in its view tube. The input cone of this lens was also $\mathrm{f} / 4.9$ and its surfaces were AR coated.

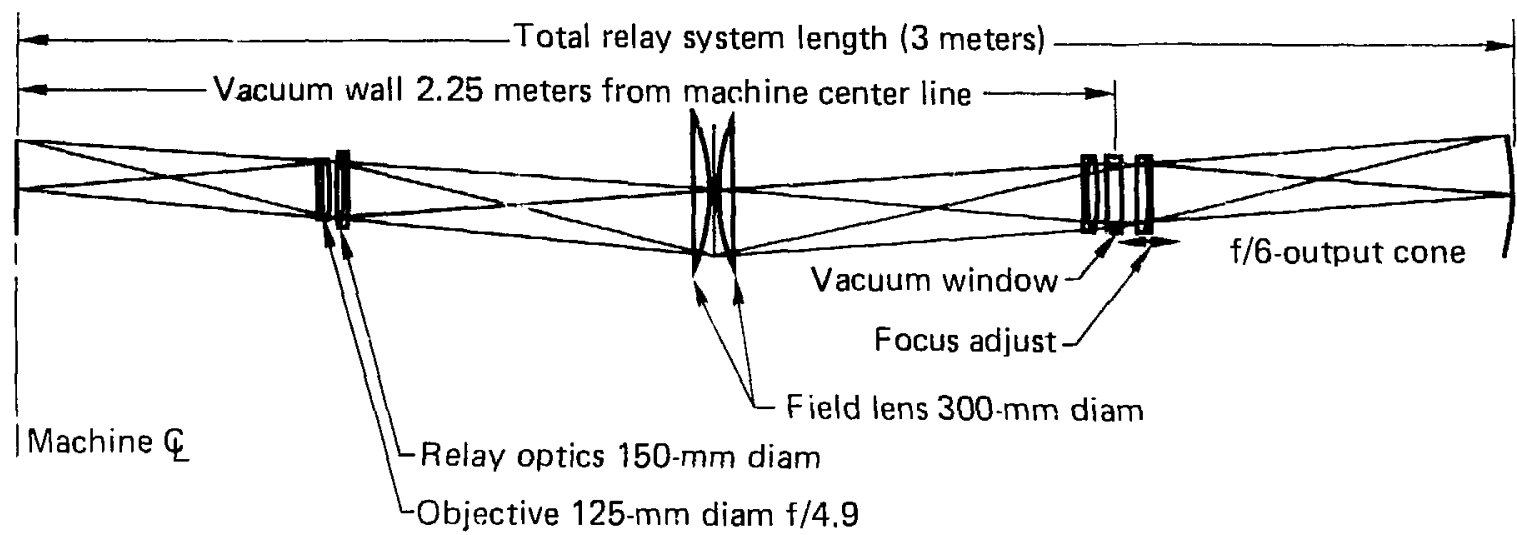

Fig. 18. Layout of the optics in the plug-system view tube. 


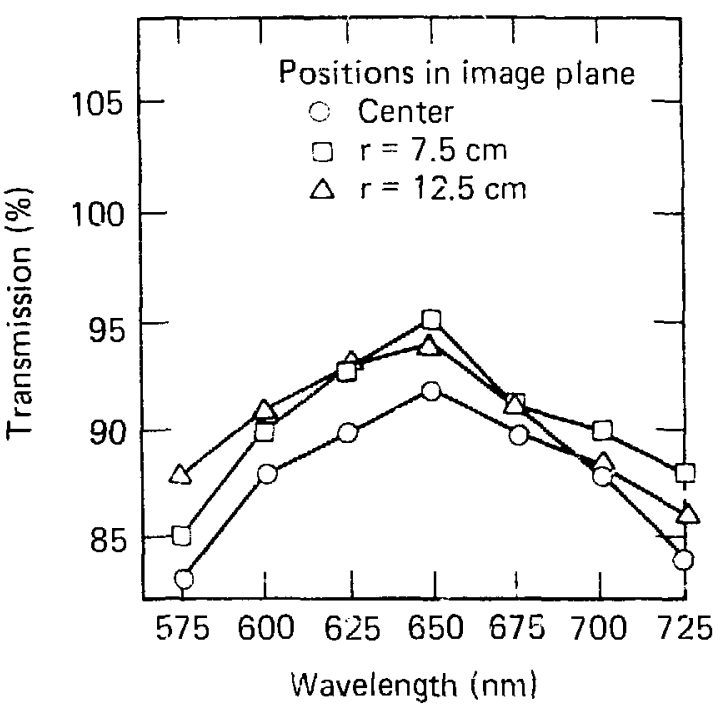

Fig. 19. Overall optical transmission of the plug-system view tube.

No special viewing dump was used in these systems. In each case the viewed tank wall was located for from the plasma. These walls were roughened to prevent specular reflections and were naturally blarkened by titanium sublimation for vacuum pumping.

\section{B. IMAGE DISTRIBUTION}

Five $1-\mathrm{cm}$ segments of the laser path are imaged by the view tube at aperture-stop slits in the image distribution box. The purpose of the aperture stop is to reduce unfocused, stray plasma radiation originating outside of the laser path. The location of these stops was set by finding the real image of marks placed at the desired positions on a string attached along the laser path. In addition, the image of the HeNe beam on the alignment flag was centered in the $R=0-\mathrm{cm}$ (plasma center) aperture stop. This was the reference that was later used to check the view tube alignment. A polarizing sheet was placed at each of these slits to discriminate against background plasma light (Section II-C).

A photograph of the plug distribution box is shown in Fig. 20; Fig. 21 is a schematic of its components. Only two polychromators were actually installed. Image separation mirrors were used to direct the desired position image through a pair of relay lenses to a polychromator. These lenses were AR-coated $125-\mathrm{mm}$-diam acromats and were set to produce a $1: 1$ image of the aperture slit on the entrance slit of the polychromator. Since the polych-omator entrance slit $(2 \mathrm{~mm}$ by $15 \mathrm{~mm}$ ) was smaller than the aperture-stop slit, it became the defining aperture of the system.

Because of the efficiency of the beam collimating and dump components (Section VI-C.), it was not necessary to use a notch filter, at the lase. wavelength, in the viewing optics. However, a high-pass filter (yellow) was placed before the polychromator entrance slit to block short wavelength plasma light that could appear in second order

A similar, but smaller, image distribution box was used in the central-cell system. It contained a single aperture-stop slit.

\section{CALIBRATION SOURCE}

A calibration lamp source, similar in both systems, was used to normalize the gains of the various $P M$ tubes and amplifiers. This light was intruduced into the viewing optical chain by a beam splitter in the distribution box (Fig. 21) and, therefore, used the polychromators and much of the same optics as the scattered light. The plasma side of the beam splitter was AR coated and its tilt direction, with respect to polarization, was chosen to minimize the losses to the scattered light coming from the plasma

A linear-filament quartz-rodide lamp, which had been calibrated for spectral emission, was the

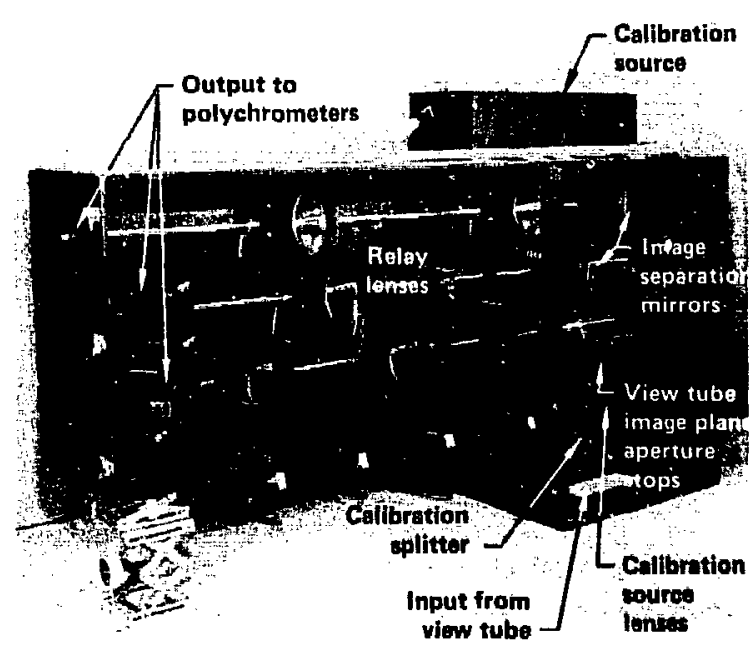

Fig. 20. Plug-system image distribuiion box. 


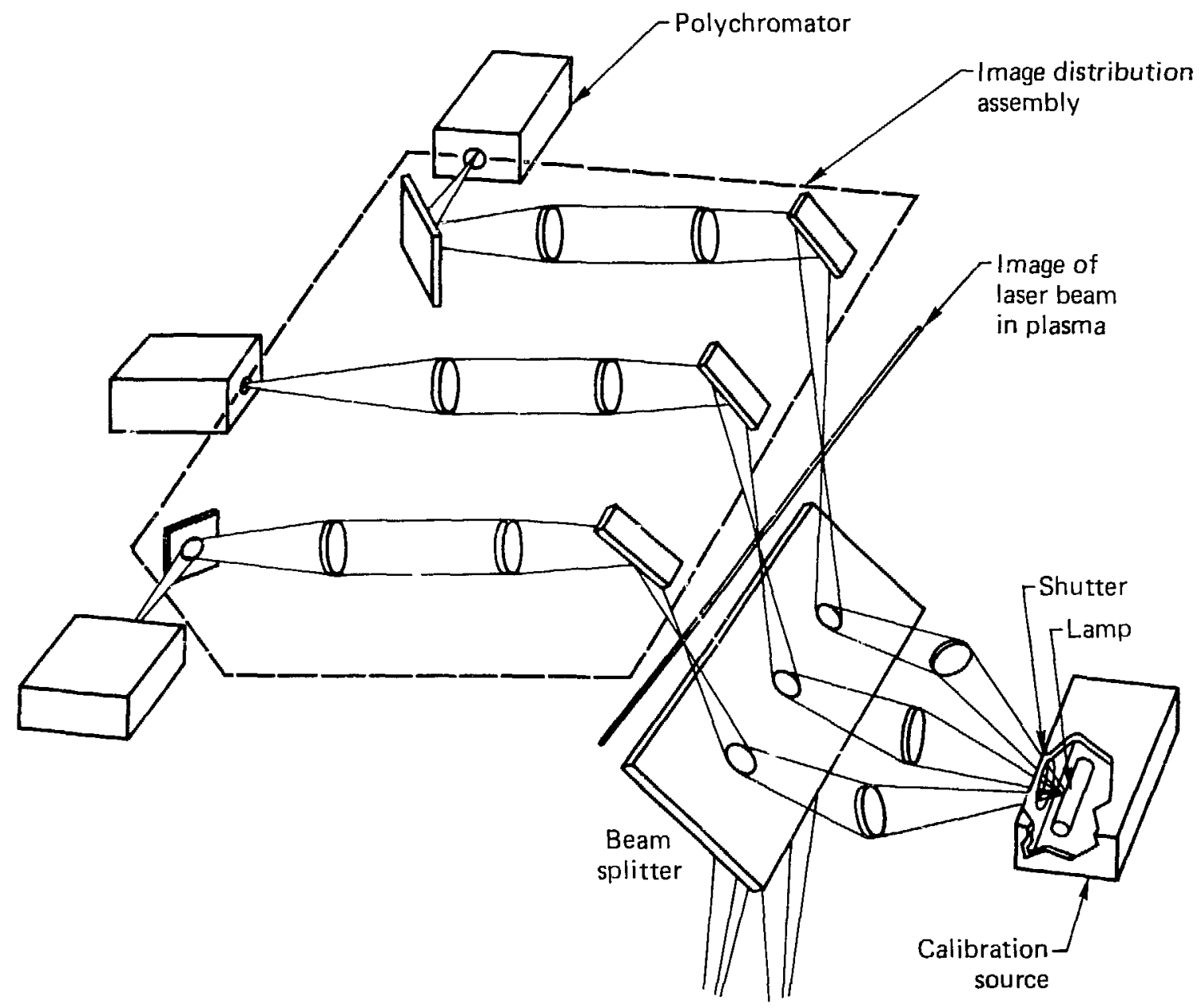

Fig. 21. Schematic diagram of the image distribution and the calibration lamp source for the plug system.

source. For spectral stability it operated continuously and a mechanical shutter was opened, after each plasma shot, for calibration of the PM channels. Separate achromatic lenses focused a portion of the lamp filament onto each aperture stop in the distribution box. A calibrated neutraldensity filter was placed below the shutter to reduce the intensity of ine calibration source to the same general level as the Thomson-scattered light.

The calibration assembly (Fig. 22) also contained a gas-discharge lamp that could be directed into the optical chain with a movable mirror. Its spectral lines were used to calibrate the polychromaturs and PM channels for wavelength.

\section{POLYCHROMATORS}

The three polychromators in these systems were commercial $600-\mathrm{mm}$ Czerny-Turner-type monochromators (Fig. 23) that we modified. The modifications were: (1) the focal length of the collimating mirror was shortened, (2) the output focusing mirror was widened, and (3) the output slit was replaced with a fiber-optic lightpipe assembly that defined the channels and interfaced the PM tubes. The design widths and spacing of the polychromator end of the light pipes are shown in Fig. 8. These light pipes had transmissions between 47 and $64 \%$. The holographic diffraction gratings in the polychromators were 


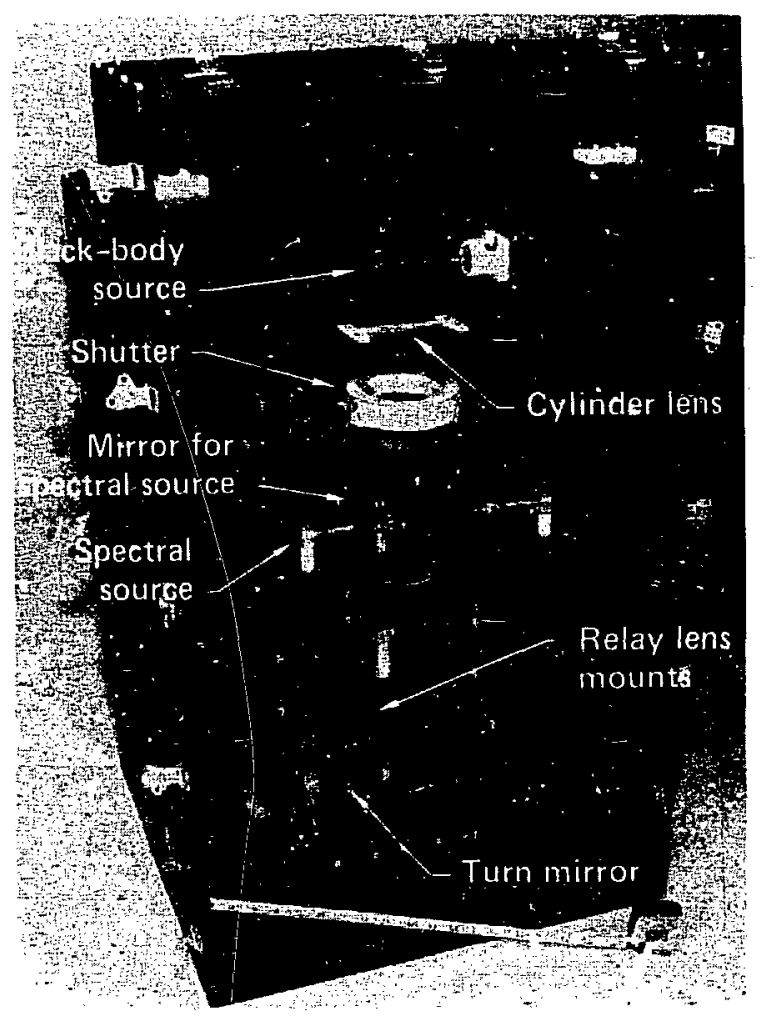

Fig. 22. Plug-system calibration lamp source.

109 by $109 \mathrm{~mm}$ and had 1200 grooves per $\mathrm{mm}$. This gave a dispersion at $7000 \AA$ of $11.5 \AA$ per $\mathrm{mm}$ at the entrance to the light pipes.

The grating rotation dial on the polychromators was calibrated for absolute wavelength at the center of the laser wavelength PM channel, No. 4, by using appropriate spectral lines from argon, neon, and hydrogen discharge tubes. With this calibrated dial and the hydrogen-alpha line $(6562.79 \AA)$, the wavelength, with respect to channel 4 , for the half-amplitude points at the edge of each PM channel was determined. For each PM channel, this absolute wavelength calibration was close to the design values.

\section{E. PHOTOMULTIPLIER TUBES.}

One of the polychromators used a set of PM tubes from the 2XIIB Thomson scattering system. These tubes had multialkali photocathodes with quantum efficiencies between 2 and $4 \%$ at $7000 \AA$ and electron gains of $10^{8}$. The other polychromators had newer tubes with transparent gallium-arsenide photocathodes and quantum efficiencies between 10 and $20 \%$. They had electron gains of only $10^{6}$ and, therefore, required amplifiers to provide suitable signal levels (Section VIII-D). To prevent saturation by background plasma light, each PM tube was gated on for $2 \mu$ s when the laser fired (Section VIII-C). The magnetic field, at the photomultiplier tube locations, was approximately 0.02 T. Each PM tube had a cylindrical magnetic shield and each set of ten tubes was enclosed in a carbon-steel box (Fig. 24). The amplifiers, where required, were also contained in these boxes. Each light pipe was mechanically clamped to the end of a PM tube and optical grease provided the light coupling.

The calibration lamp and a set of calibrated neutral-density filters were used to determine the saturation level of each PM tube. The high voltage on each PM tube was adjusied so the tube always operated below this limit.

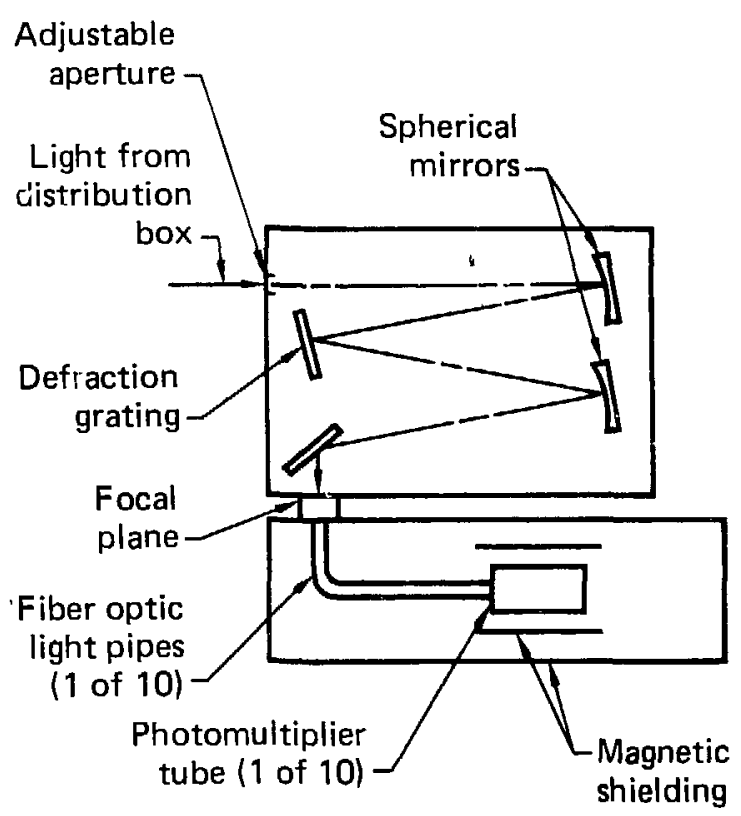

Fig. 23. Schematic drawing of a polychromator and photomultiplier tube assembly. 




Fig. 24. Photomultiplier tube set.

\section{ELECTRONICS AND SIGNAL HANDLING}

\section{A. GENERAL OPERATION AND TIMING}

A functional block diagram for the automatic operation of the TMX central-cell system is shown in Fig. 25. The pulses to charge the laser, open the shutters, and fire the laser came from the master TMX timing system. The generation of the PMtube gates and the operation of the calibration lamp shutter was controlled by the autocalibrator (Section VIII-B). The PM signals were interfaced to a computer (Section VIII-E) for automatic analysis and could also be recorded with oscilloscope cameras for hand analysis. io avoid reflection problems, all pulse and signal coax cables were operated as $50-\Omega$ terminated lines.

The firing sequence began with a $t=-5-\mathrm{ms}$ pulse from the TMX timer. The first digital delay was set to compensate for the delays built into the laser so that it would fire at $t=0$ if the second digital delay was sef to zero. This sornnd delay selected the meaburement time during the plasma shot: typically $t \quad 10 \mathrm{~ms}$. The typical length of a plasma shot was $25 \mathrm{~ms}$. The pulse to fire the laser Q-switch triggered a section in the autocalibrator that generated the $\delta-\mu s$-wide PM tube gate. Cable delays were added to fine tune this timing and approximately center the laser firing time in this gate time.

The output signal from each PM tube was split five ways, one to a scope and four to a computer, by a fan-out that preserved the $50-\Omega$ termination in each direction. This fan-out unit also contained linked 50- $\Omega \mathrm{T}$-pad-type attenuators on each output except the one for the scope. The separation of the computer part of each PM signal into four parts provided separate measurements of the Thomsonscattered signal (prompt) and three samples of the plasma radiation (Section IV-C) signal (delayed $1,2,3)$ on each shot. The three plasma radiation samples allowed rejection, by the omputer, of noise spikes on this radiation.

The four computer outputs from each PM channel went to separate analog to-digital (A/D) converter modules that were grated at different times. These modules were commercial 12-channel 


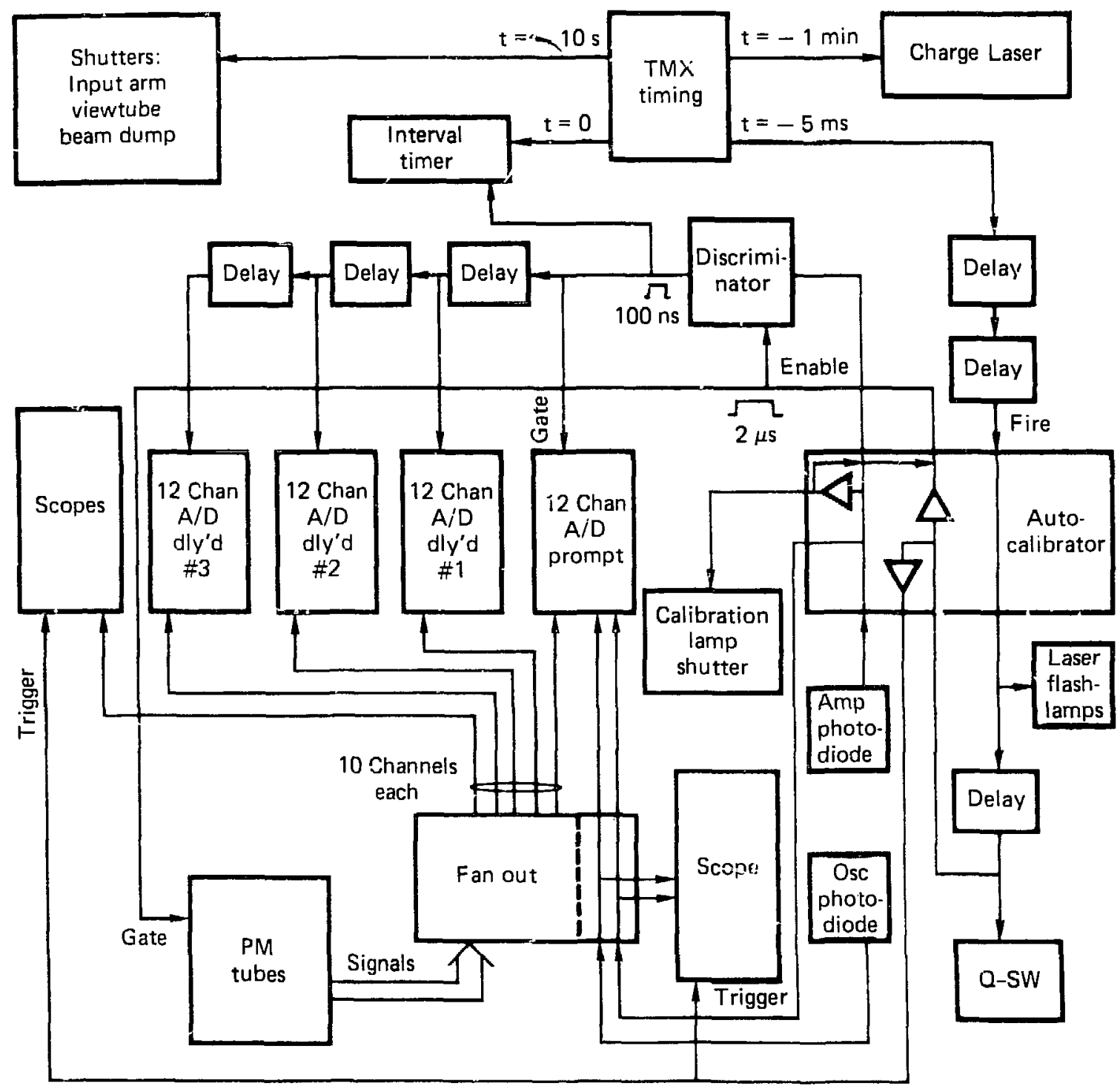

Fig. 25. Electronics block diagram for the central-cell Thomson scattering system.

charge-sensitive A/D converters with a 32-deep memory on each channel. Each $A / D$ channel effectively integrated and stored the signal received during its gate time. The 100-ns-wide gate for the prompt module was generated by a discriminator. This commercial discriminator module was triggered by the signal from the photodiode that measured the total laser energy (Section VI-A). Cable delays were used to center the arrival of the scattered-light signal within the time of the prompt module gate. The gate pulses to the modules measuring the plasma radiation samples were further delayed by $100 \mathrm{~ns}$ each. With this arrangement the scattered signal and three plasma radiation sample signals for each PM channel were stored and could be read by the computer at a later time. The output of the laserenergy photodiodes were also recorded on channeis of the prompt $\mathrm{A} / \mathrm{D}$ module. The discriminator setting, and the enable pulse (from the PM gate) to the discriminator was to prevent noise pulses from triggering this unit at the wrong time.

The plug system was the same as described above except that it had a second set of four A/D modules for the second polychromator and PM tube set. The gates for these modules were the same as those for the first set. 


\section{B. AUTOCALIBRATOR}

The autocalibrator unit generated the PM gates and oscilloscope triggers. However, its main function was to control the relative calibration of each PM channel. At approximately $350 \mathrm{~ms}$ following the laser firing (the plasma shot was over) it gated the A/D modules to msasure the zero level of each AID channel. Then it opened the calibration lamp shutter and, at approximately $150-\mathrm{ms}$ intervals, it gated the PM tubes and the $A / D$ modules fifteen times. This sequence produced fifteen measurements of the calibration lamp signal on each A/D channel (prompt and delayed) that were also stored in their memory. These provided a direct measurement of the photon statistics of each PM tube and was used by the computer to compute a standard deviation for each channel (Section IX-A). The average of these measurements, for each channel, was the gain normalization factor for all the PM output signals of that channel. This calibration was performed for each plasma shot and removed any errors that would be caused by drifts in gains. For these calibrations, the time of the prompt A/D module gate within PM gate was set to be the same as the time of the laser firing within this gate in case the gain of any PM tube varied during its gating.

\section{PHOTOMULTIPLIER-TUBE GATING}

To gate the PM tubes (Section VII-E) a speciai tube base containing the dynode voltage divider string with gating circuitry was designed and constructed. The schematic diagram for the high-quantum efficiency tubes (Section VIII-E) is shown in Fig. 26. The gating circuitry for the lower-quantum efficiency tubes was functionally similar, but with certain modifications to accommodate differences in tube properties. The resistor chain divides the high voltage $(-3500 \mathrm{~V})$ betwein each of the tube elements while the capacitators store sufficient charge to keep the dy node voltages constant during the 2 - $\mu 5$-gated period when $\because$ current drain is relatively high.

Gider normal operation, both switches are in $\cdots=z: d$ position. In this 1 ode, with no gate $\because=\because$ rodes 4 and 8 are at a more negative $\because \cdots . . . .20 n$ dynodes 3 and 7 , respectively. This $\because: \because: \cdots:=:$ rectric field in the tube that opposes $\because, \ldots, \cdots,:-\cdots r n s$ and effectively turns off the

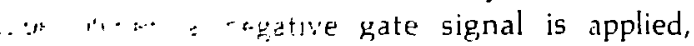
$\ldots \cdots, \cdots, \cdots, \cdots, Q]$ and $Q 2$, the proper voltages are applied to allow current to flow in the PM tube. Proper operation of this gating circuitry is dependent on the characteristics of the switching transisturs. These particular PN!P transistors were chosen for their high emitter-collector standoff voltage. Capacitors $C_{1}$ and $C_{2}$ were selected to accommodate the base resistance of the transistor and the drive capabilities of the gate signal.

When calibrating the grating rotation dial on the polychromators (Section VII-D), the PM tubes were operated in the continuous mode with the switches in the $C W$ position. In this way the gating circuitry is bypassed. To limit the average anode current to its allowable level, in this mode of operation, a high impedance $(\sim 1 \mathrm{M} \Omega)$ series element was inserted between the anode and input of the measurement instrument. For norinal uperation in the gated mode, the anode was terminated in $50 \Omega$.

\section{PHOTOMULTIPLIER-TUBE AMPLIFIERS}

For the sets of PM tubes having a gain of only $10^{\circ}$ (Section VII-E), an additional amplifier was added between the PM tube and the A/D converters. The linear amplifier used was a 12-channel NIM packaged unit with fixed gain of 10 and having a 200-MHz bardwidth. Two of the lowerlevel channels required gains of 100 that was achieved by connecting two of the amplifie: channels in series. Although we used a commercially available NIM module we did not install it in a NIMBIN, but instead made a special installation inside of the PM tube enclosure box.

\section{E. COMPUTER INTERFACE}

After the shot, the data stored in the memory of the A/D converter modules were transferred to the TMX computer svstem. Figure 27 is a block diagram of the interface system. The photoisolator modules were the standard units used on all diagnostic interfaces to the TMX computer system. In collecting the data, we utilized the addresss scan mode of the cratc controller interface. This feature allows block transfers of data initiated by a single computer command. A CAMAC command with $N=1, A=0, F=2$, and a word count of 144 would cause the controller to transfer the fir it word of the 32-deep ADC menury from each 




Fig. 26. Schematic of a photomultiplier tube base and gating circuit. 

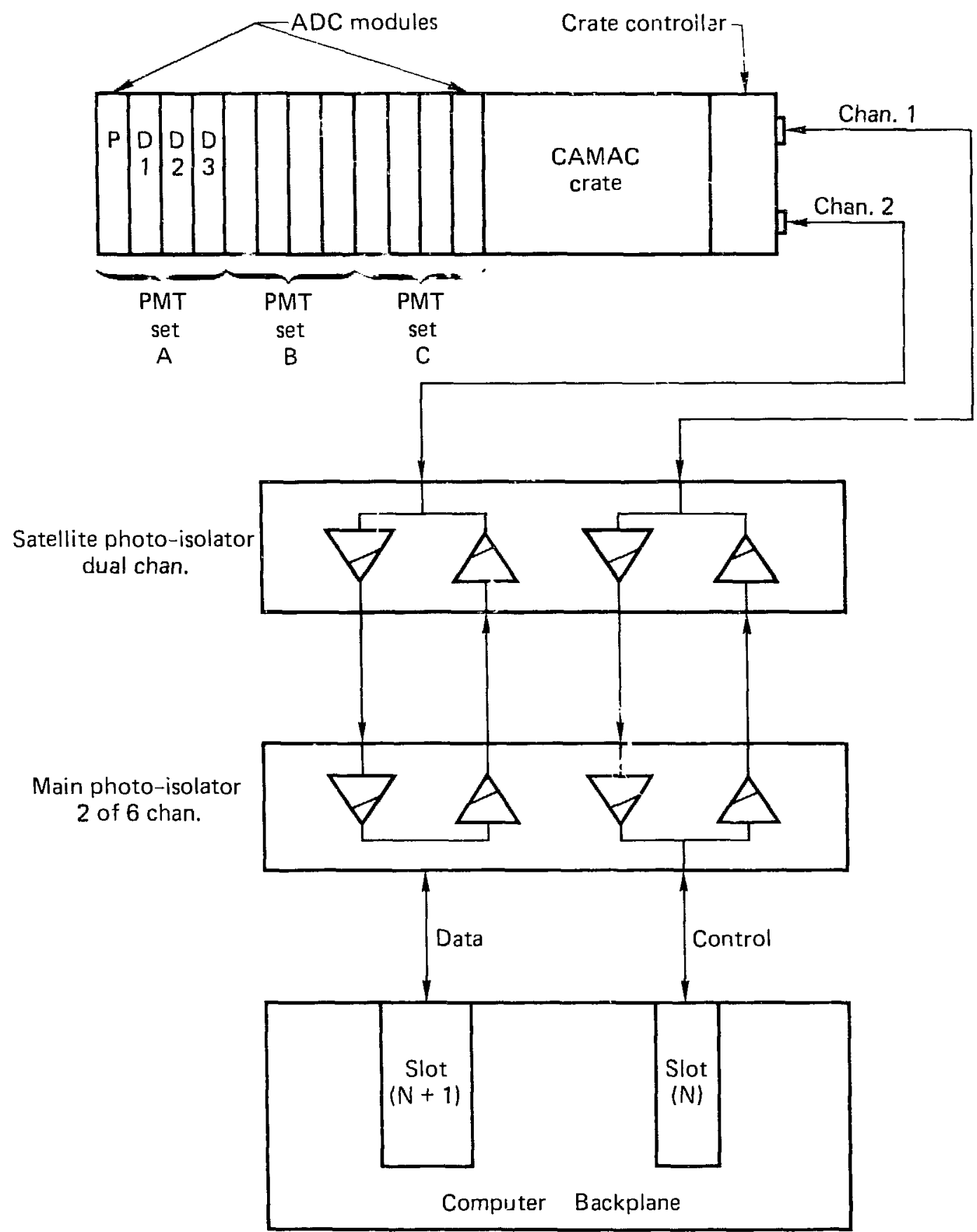

Fig. 27. Block diagram of the computer interface system. 
channel $(A=0-11)$ of each module $(N=1-12)$. Using the $F=2$ command for the ADC modules n'ould clear the output word of the first-in-firstout (FIFO) memory and shift out the next word for the following read command. In this way, by reissuing the same command, the next layer of 144 data words would be transferred. This was done 17 times to read out the data from the 17 gated in'ervals of each PM tube (Section VIII-B).

\section{DATA REDUCTION}

\section{A. ERROR ANALYSIS}

The total photon flux (F) into a given PM tube for a Thomson scattering measurement, can be expressed as

$$
\mathrm{F}=\mathrm{CV}
$$

where $V$ is the output read by the computer from the charge-sensitive A/D converter (Section VIIl-A) and is proportional to the number of photoelections emitted by the tube photocathode. The calibration constant $C$ includes the photocathode quantum efficiency and all of the gain factors for that channel. A relative $C$ for each channel is miasured after each plasma shot by using the calibration lamp (Sections VII-C and VIII-B) and can be written as

$$
C=\frac{F_{c}}{\bar{V}_{c}}
$$

where:

$$
\begin{aligned}
\mathrm{F}_{\varepsilon}= & \text { calibration lamp photon flux into } \\
& \text { the PM tube and } \\
\bar{V}_{\mathrm{c}}= & \text { the average of the A/D outputs } \\
& \text { from the fifteen calibration } \\
& \text { measurements. }
\end{aligned}
$$

While $\mathrm{F}_{\mathrm{c}}$ is not absolutely calibrated,

$$
\frac{F_{\mathrm{C}}}{W} \mathrm{~S}=\mathrm{K}
$$

where $K$ is constant for all of the rhannels of a polychromator. Here $W$ is the wavelength width of the channel and $S$ is the relative spectral output per wavelength for the calibration lamp. Since all PM output signals are referenced to the calibration lamp intensity, the calibration of the system for density (Section II-D) effectively calibrates this $\mathrm{K}$ in terms of plasma density.

From Eq. (12), the percent error in F may be expressed by 23

$$
\left(\frac{\sigma_{F}}{F}\right)^{2}=\left(\frac{\sigma_{C}}{C}\right)^{2}+\left(\frac{\sigma_{V}}{V}\right)^{2}
$$

where $a$ is a standard deviation. Because the photon-electron conversion in a PM tube is governed by Poisson statistics, and assuming that the percent error of a single scattering measurement is approximately the same as the percent error in the calibration lamp measurements, a constant can be obtaned

$$
\frac{\sigma_{V}}{\sqrt{V}} \simeq \frac{\sigma_{V_{c}}}{\sqrt{\bar{V}_{c}}}=\text { constant }
$$

From Eq. (13) the percent error in $C$ can be written as $^{23}$

$$
\frac{\sigma_{\mathrm{c}}}{C}=\frac{\sigma_{\bar{V}_{c}}}{\bar{V}_{c}}=\frac{\sigma_{V_{c}}}{\sqrt{N_{c}} \frac{1}{\bar{V}_{c}}}
$$

where $N_{c}$ is the number of calibration measurements. By using Eqs. (16) and (17), Eq. (15) becomes

$$
\left(\frac{\sigma_{F}}{F}\right)^{2}=\frac{\left(\frac{\bar{V}_{c}}{V}+\frac{1}{N_{c}}\right)}{\left(N_{c}-1\right)}
$$

$$
\times\left[\frac{\sum_{i} V_{c i}^{2}}{\bar{V}_{c}^{2}}-N_{c}\right]
$$

where $\mathrm{i}$ is an index on the calibration lamp measurements.

\section{B. COMPUTER ANALYSIS}

The computer reduction of Thomson scattering data was performed between plasma shots by 




Fig. 28. Typical TMX Thomson-scattering data reduction. 
the TMX diagnostic computer system. ${ }^{24}$ Background shots (no plasma present) were regularly taken on TMX for various diagnostic systems. These were used by the Thomson scattering systems to record any stray laser light (Section II-C) received by a PM channel. Following each background and plasma hot the computer read, and stored in arrays, the data from the memory of all the AlD modules (Section VIII-E).

For each PM tube, the appropriate A/D channel baseline was subtracted from each separate output signal (prompt, delayed, and calibration). The computer then applied the averages of the sets of calibration lamp measuremen is as a gain normalization factor to the corresponding prompt and delayed signals and also calculated the errors in each ot these signals from Eq. (18). Any delayed signal that was greater than $2 \sigma$ above the lowest of the three delayed signals, was considered to be a noise spike and was rejected. The average of the remainung delayed signals was subtracted from the prompt signal and the result was normalized to the total laser power.

The computer performed all of the above calculations on both the plasma shot and the appropriate background shot. The value from the backgrnund shot (stray laser light) was subtracted from the value for the plasma shot and the final result was proportional to the Thomson scattered photon flux that entered the PM tube of that channel. The total error for each channel was calculated from the errurs of each contributing signal as

$$
\sigma^{2}=\sum_{i} \sigma_{i}^{2}
$$

where $\mathrm{i}$ indexes prompt and delayed for buth the plasma and background shots.

From these results for each PM channel, the computer fits a two-parameter $\left(T_{e}, n_{e}\right)$ Gaussian to the points by the grid-search method of least squares. ${ }^{23}$ Each point used in the fit was weighted by it s calculated error $\sigma$ from Eq. (19). Fits at $+o$ and $-o$ from the best fit, were also obtained to provide the total orrors in the measurement of $T_{e}$ and $n_{e}$. A typical output from this computer analysis is shown in Fig. 28. When this data was taken, two PM tubes (channels 1 and 9) were saturating and they, along with the laser wavelength (channei 4 ), were not included in the analysis. The error bars on each point show the error determined from Eys. (18) and (19).

\section{RESULTS}

Thomson scattering measurements from some of the TMX experiments are given in Figs. 29-34. Figure 29 shows a shot-by-shot radial profile of the electron temperature in the east plug for gas-box operation of TMX.16, 17 This profile is relatively flat out to $R=5 \mathrm{~cm}$, then is down by a factor of 2 or 3 at $10 \mathrm{~cm}$. The comparison of simultaneous east-plug $(R=0)$ and central-cell electron temperatures in Fig. 30 shows that the central-cell $T_{e}$ is generally comparable to that in the east plug. The existence of central-cell temperatures of $50 \mathrm{eV}$ and above indicates that there are no large energy losses due to impurities trapped in the central cell. This plot also shows that because these temperatures often differ by a factor of 2, there can be some isolation between the central-cell and end-plug electrons. The smaller error bars on the central-cell temperatures are due to the higher quantum efficiency of the central-cell PM tubes (see Section II-C).

On the day that the data in Fig. 30 were obtained, one of the coils in the west plug developed an air leak. However, even with the resulting low temperatures the electrons were isolated. The radial profiles of $T_{L}$ at $R=0$ and $5 \mathrm{~cm}$ in the east plug and at $R=0$ in the central cell for these same shots are shown in Fig. 31. It can be seen that the temperatures generally track each other. The point density profiles for these shots a re given in Fig. 32. For comparison, the densities obtained by unfolding line-density measurements from beam attenuation ${ }^{24}$ (Gaussian fits) are also plotted. The rise in the central-cell $n_{e}$ due to the air leak in the west plug can be seen. The east-plug densities are little affected by this rise and are relatively flat out to a $5 \mathrm{~cm}$ radius. The higher central-cell densities are associated with the lower electron temperatures. These data show that poor vacuum conditions can result in lower $T_{c}$ - The background gas pressure during a shot may also be affected by wall conditions.

In Fig. 33, the radial plug potentials $(\phi)-$ measured by electrostatic end-loss analyzers ${ }^{24}$ are compared with the radial electron temperatures in the east plug measured on the same series of shots. Here, measurements from several shots 
at tach position are averaged and the error bars are the standard deviations of the measurements. These data are from central-cell gas putier operation of TMX. It can be seen that the ratio of $\phi$ to $T_{*}$. for the plug is approximately five.

The central-cell potentials were measured by the heavy-ion-beam-probe (HIBP). ${ }^{24}$ Figure 34 shows a comparison of some of these measurements with electron temperature measurements in both the central cell and east plug. Here, the central-cell potentials are plotted at an equivalent magnetic field line position in the midplane of the plug. The central-cell potential and the plug electron temperture have similar profiles.

Fig. 29. A shot-by-shot radial profile of the electron temperature in the east plug.
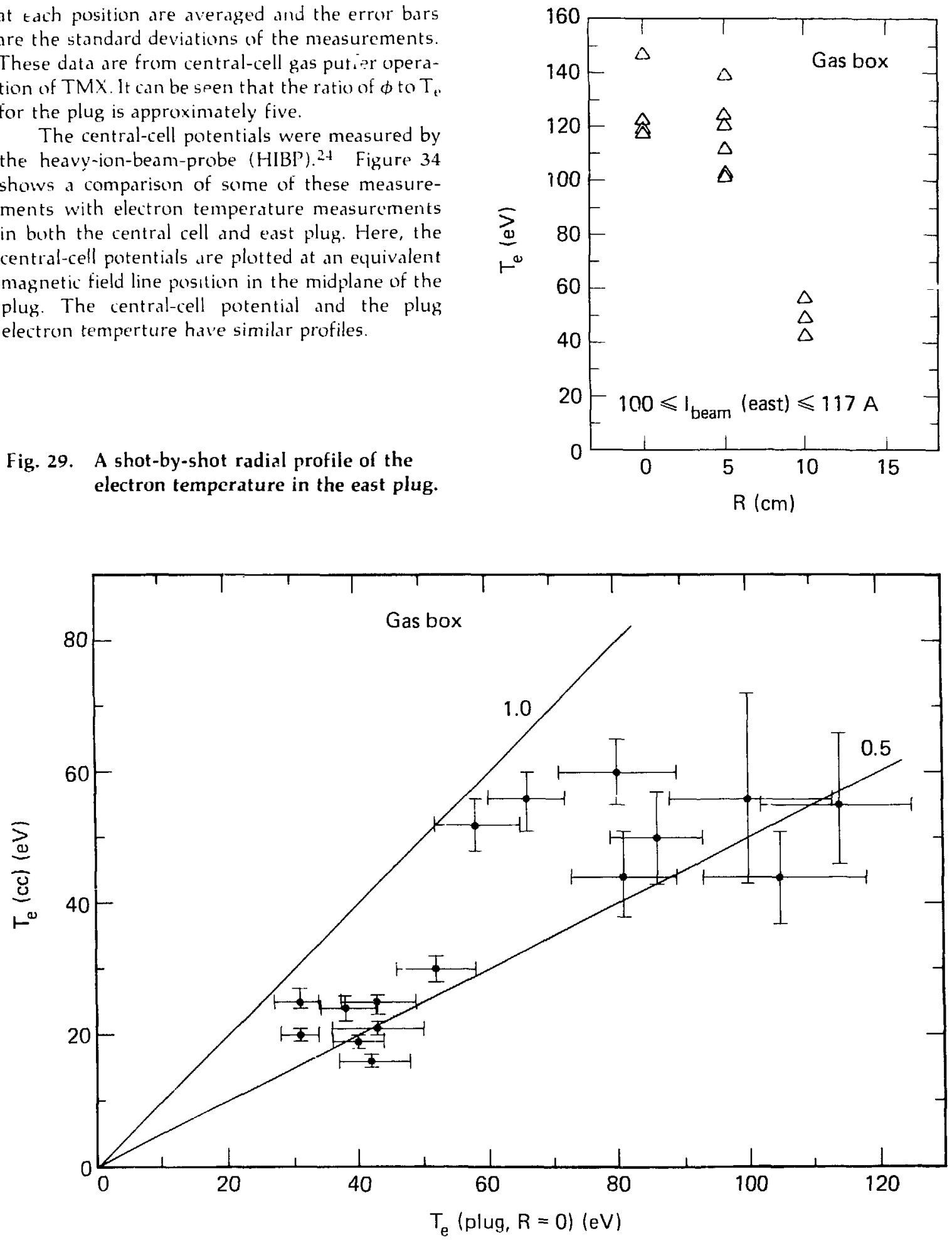

Fig. 30. A cor arison of simultaneous central cell and east plug $(R=0)$ electron temperatures. The line represent $T_{e}$ (central cell) equals $T_{e}$ (plug), and $T_{e}$ (central cell) equals $0.5 T_{e}$ (plug). The group of lower temperatures is associated with a vacuum leak in the west plug. 
Fig. 31. Electron temperatures at two radii in the TMX east plug compared to central-cell electron temperatures. The solid lines connect simultaneous measurements. These data are from the same shots as Fig. 30.
Fig. 32. Plasma densities at two radii in the TMX east plug compared to centralcell densities. Also shown are densities obtained from beam attenuation measurements. These data are for the same shots as Fig. 30.
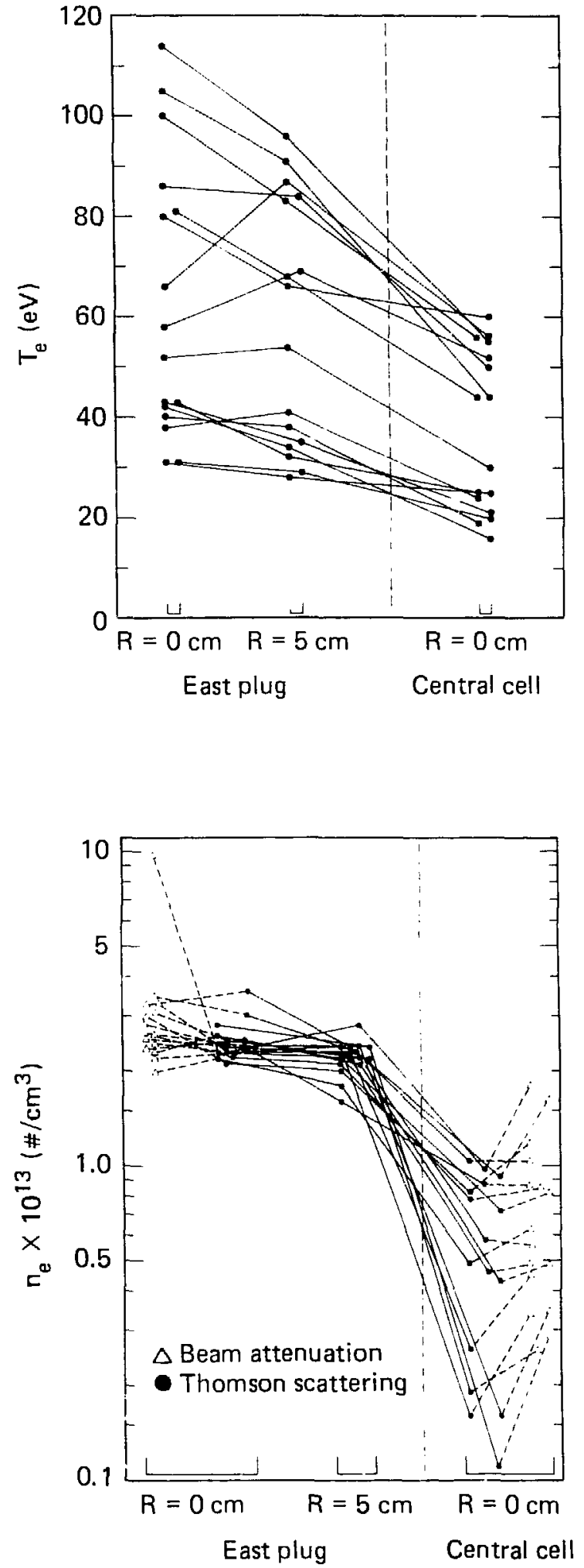




Fig. 33. A comparison of radial plug potentials with plug radial electron temperatures. Potentials obtained with electrostatic end-loss analyzers. - refers to $\phi$, west end-loss analyzer, - to the $\phi$, east end-loss analyzer, and $\Delta$ to $T_{e}$, east plug.

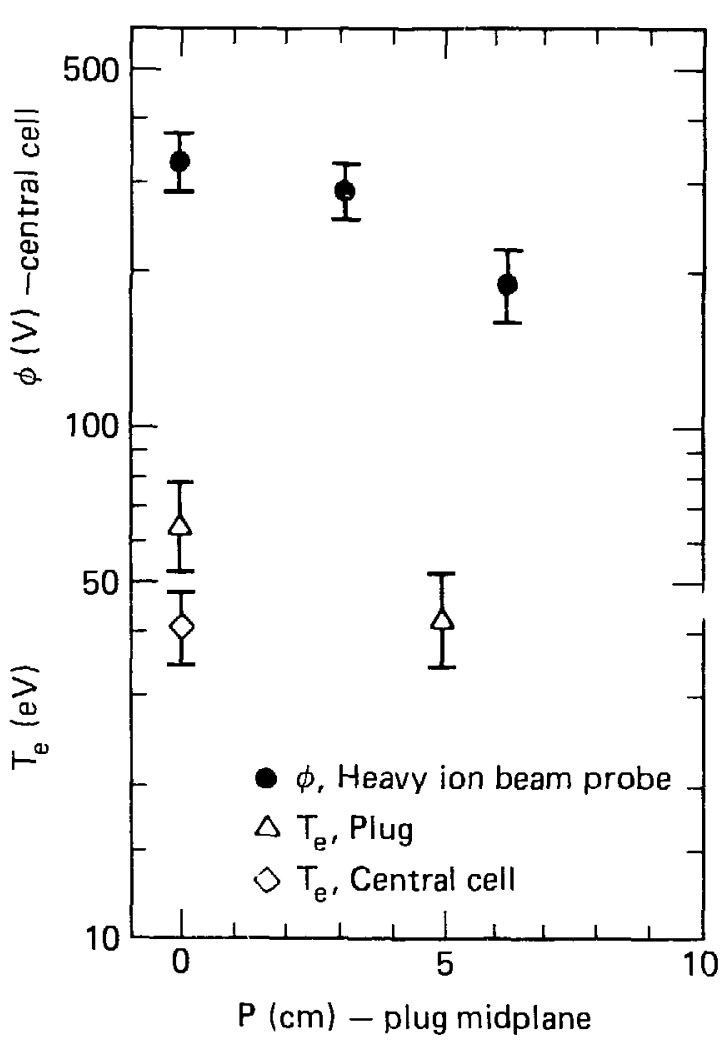

Fig. 34. The central-cell radial-potential profile, measured by the heavy-ionbeam probe, compared with centralcell and east-plug electron temperatures.

\section{ACKNOWLEDGMENTS}

The authors wish to thank Sherman Jenkins and John Jolly for their contribution to the assembly, operation, and maintenance of these systems. We also appreciate the help of Cecil Howard with the various optical tests and alignments.
This work was performed under the auspices of the U.S. Depar ıment of Energy by the Lawrence Livermore National Laboratory under contract number W-7405-ENG-48. 


\section{REFERENCES}

1. E. T. Gerry and D. J. Rose, "Plasma Diagnostics by Thomson Scattering of a Laser Beam," I. Appl. Phy/s. 37, 2715 (1960).

2. M. Daehler and F. L. Ribe, "Cooperative Light Scattering from A-Pinch Plasmas," Phys. Rev. 161, 117 (1976).

3. M. J. Forrest, N. J. Peacock, D. C. Robinson, V. V. Sannikov, and P. D. Wilson, Metsurement of the Plasma Paramtitrs in Tokamak T3-A by Thomson Sattering. Culham Laboratory, Abingdon, U.K., CLM-R 107 (1970).

4. M. Murakam, W. R. Wing, J. S. Culver, and P. H. Edmonds, Thomson Sattering Marasurements in ORMAK, Oak Ridge National Laboratory, Oak P'tge, TN, ORNL-TM-4354 (1973).

5. T. C. Simonen, The 2XIIB Thomson Sattering System. Lawrence Livermore National Laboratory, Livermore, CA, UCRL-51408 (1973).

o. K. A. Gerber, Laser Sattering System on the' NRL Triton Experiement, Naval Research Laboratory, Washington, DC, NRL-2818 (1974).

7. A. Ascoli-Bartoli, A. Brusadin, A. Cecchini, A. DeAngelis, F. Garosi, E. Grilli, R. Marchetti, R. Marconero. L. Pieroni, and S. E. Segre, The 900 Thomson Sattering Apraratus for the Alcator Device, Associazione EURATOM-CNEN sulla Fusione, Centro Gas Ionizzati, Frascati, Italy (1975).

8. J. D. Sethian and C. A. Ekdahl, "Cornell Thomson Scattering Systern," Rer. Sii. Instrim. 49, 729 (1978).

9. N. Bretz, D. Dimock, V. Foote, D. Johnson, D. Long and E. Tolnas, "Multichannel Thomsor, Scattering Apparatus," Appl. Opt. 17, 192 (1978).

10. P. E. Phillips and P. Nielson, "Thomson Scattering ai: $10^{\circ}$ on the Texas Turbulent Torus," Plasms Phys. 20, 1265 (1978).

11. R. Prentice, The DITE Tokamak Multipulse Thomson Sattering Sustem. Culham Laboratory, Abingdon, U.K., CLM-R 179 (1978).

12. J. Lasalle and P. Platz, "Multichannel Thomson Scattering System for Tokamak TFT Based on Two-Detector Spectrum Analyzers," Apll. Opt. 18, 4124 (1979).

13. M. I. Forrest, P. A. Jones, N. J. Peacock, R. Prentice, A. C. Selden, C. I. Walker, and S. Ward, Spacial Thumsam Santtering System. Culham Laboratory, Abingdon, U.K., Jet Design Study 8.2 (1980).

14. A. M. Frank, MFTF Thomson Santturing: A System Stuty, Lawrence Livermore National Laboratory, Livermore, CA, UCRL-53051 (1980).

15. L. Bighel, J. Cobble, Electron Hoating and Continument Mensurments in EBT-S Using Thomson Scattring. Oak Ridge National Laboratory, Oak Ridge, TN, ORNL-TM-7437 (1980).

16. T. C. Simonen et. al., "Plasma Confinement Experiments in the TMX Tandem Mirror," in Pror. Intern. Conf. on Plasma Physics and Controlled Nuclear Fusion Rusearch, sth, Brussels, 1980 (IAEA, Vienna, 1981).

17. TMX Group, Summary of Results from the Tandem Mirror Experiment (TMX), Lawrence Livermore National Laboratory, Livermore, CA, UCRL-53120 (1981).

18. H. J. Kunze, "The Laser as a Tool for Plasma Diagnostics," Plnsma Dingnostics, W. Lochte-Holtgreven, Ed. (North Holland Publishing Company, Amsterdam, 1968), p. 550.

19. J. Sheffield, Plasma Scattering of Electromagnetic Radintion (Academic Press, New York, 1975).

20. M. Mattioli and R. Papoulas, "Analysis of Light Scattered from Relativistic Plasmas," Plasma Phys. 17, 165 (1975).

21. T. Matoba, T. Itagaki, T. Yamauchi, and A. Funahashi, "Analytical Approximations in the Theory of Relativistic Thomson Scattering for High Temperature Fusion Plasma," Jap. I. Appl. Phys. 18, 1127 (1979).

22. B. A. Jacoby, T. M. York, and R. A. Mollo, A Doubled Nit-Glass Laser System for Incoherent Thomson Senttering from Low Density Plasmas, Pennsylvania State University, Staie College, Pa, COO-4020-8 (1979).

23. P. R. Bevington, Data Retuction and Erro, Analysis for the Physical Sciences (McGraw-Hill, New York, 1969).

24. TMX Group, Summary of Results from the Tandem Mirne Expriment (TMX), Diagnostic Appendix, Lawrence Livermore Naticnal Laboratory, Livermore, CA, UCRL-53120 (1981). 